\title{
Computation of eigenvalues of discontinuous dirac system using Hermite interpolation technique
}

Mohammed M Tharwat ${ }^{1,2^{*}}$ and Ali H Bhrawy ${ }^{1,2}$

\author{
* Correspondence: \\ zahraa26@yahoo.com \\ 'Department of Mathematics, \\ Faculty of Science, King Abdulaziz \\ University, Jeddah, Saudi Arabia \\ Full list of author information is \\ available at the end of the article
}

\begin{abstract}
We use the derivative sampling theorem (Hermite interpolations) to compute eigenvalues of a discontinuous regular Dirac systems with transmission conditions at the point of discontinuity numerically. We closely follow the analysis derived by Levitan and Sargsjan (1975) to establish the needed relations. We use recently derived estimates for the truncation and amplitude errors to compute error bounds. Numerical examples, illustrations and comparisons with the sinc methods are exhibited.

Mathematical Subject Classification 2010: 34L16; 94A20; 65L15.

Keywords: Dirac systems, Hermite interpolations, transmission conditions, discontinuous boundary value problems, truncation and amplitude errors, sinc methods
\end{abstract}

\section{Introduction}

Let $\sigma>0$ and $\mathrm{PW}_{\sigma}^{2}$ be the Paley-Wiener space of all $L^{2}(\mathbb{R})$-entire functions of exponential type type $\sigma$. Assume that $f(t) \in \mathrm{PW}_{\sigma}^{2} \subset \mathrm{PW}_{2 \sigma}^{2}$. Then $f(t)$ can be reconstructed via the sampling series

$$
f(t)=\sum_{n=-\infty}^{\infty}\left[f\left(\frac{n \pi}{\sigma}\right) S_{n}^{2}(t)+f^{\prime}\left(\frac{n \pi}{\sigma}\right) \frac{\sin (\sigma t-n \pi)}{\sigma} S_{n}(t)\right],
$$

where $S_{n}(t)$ is the sequences of sinc functions

$$
S_{n}(t):= \begin{cases}\frac{\sin (\sigma t-n \pi)}{(\sigma t-n \pi)}, & t \neq \frac{n \pi}{\sigma}, \\ 1, & t=\frac{n \pi}{\sigma} .\end{cases}
$$

Series (1) converges absolutely and uniformly on $\mathbb{R}$ (cf. [1-4]). Sometimes, series (1) is called the derivative sampling theorem. Our task is to use formula (1) to compute eigenvalues of Dirac systems numerically. This approach is a fully new technique that uses the recently obtained estimates for the truncation and amplitude errors associated with (1) (cf. [5]). Both types of errors normally appear in numerical techniques that use interpolation procedures. In the following we summarize these estimates. The truncation error associated with (1) is defined to be

\section{苗




$$
R_{N}(f)(t):=f(t)-f_{N}(t), \quad N \in \mathbb{Z}^{+}, \quad t \in \mathbb{R},
$$

where $f_{N}(t)$ is the truncated series

$$
f_{N}(t)=\sum_{|n| \leq N}\left[f\left(\frac{n \pi}{\sigma}\right) S_{n}^{2}(t)+f^{\prime}\left(\frac{n \pi}{\sigma}\right) \frac{\sin (\sigma t-n \pi)}{\sigma} S_{n}(t)\right] .
$$

It is proved in [5] that if $f(t) \in \mathrm{PW}_{\sigma}^{2}$ and $f(t)$ is sufficiently smooth in the sense that there exists $k \in \mathbb{Z}^{+}$such that $t^{k} f(t) \in L^{2}(\mathbb{R})$, then, for $t \in \mathbb{R},|t|<N \pi / \sigma$, we have

$$
\begin{aligned}
\left|R_{N}(f)(t)\right| \leq T_{N, k, \sigma}(t):= & \frac{\xi_{k, \sigma} E_{k}|\sin \sigma t|^{2}}{\sqrt{3}(N+1)^{k}}\left(\frac{1}{(N \pi-\sigma t)^{3 / 2}}+\frac{1}{(N \pi+\sigma t)^{3 / 2}}\right) \\
& +\frac{\xi_{k, \sigma}\left(\sigma E_{k}+k E_{k-1}\right)|\sin \sigma t|^{2}}{\sigma(N+1)^{k}}\left(\frac{1}{\sqrt{N \pi-\sigma t}}+\frac{1}{\sqrt{N \pi+\sigma t}}\right),
\end{aligned}
$$

where the constants $E_{k}$ and $\xi_{k, \sigma}$ are given by

$$
E_{k}:=\sqrt{\int_{-\infty}^{\infty}\left|t^{k} f(t)\right|^{2} d t}, \quad \xi_{k, \sigma}:=\frac{\sigma^{k+1 / 2}}{\pi^{k+1} \sqrt{1-4^{-k}}} .
$$

The amplitude error occurs when approximate samples are used instead of the exact ones, which we can not compute. It is defined to be

$$
\begin{aligned}
\mathcal{A}(\varepsilon, f)(t)=\sum_{n=-\infty}^{\infty} & {\left[\left\{f\left(\frac{n \pi}{\sigma}\right)-\tilde{f}\left(\frac{n \pi}{\sigma}\right)\right\} S_{n}^{2}(t)\right.} \\
& \left.+\left\{f^{\prime}\left(\frac{n \pi}{\sigma}\right)-\tilde{f}^{\prime}\left(\frac{n \pi}{\sigma}\right)\right\} \frac{\sin (\sigma t-n \pi)}{\sigma} S_{n}(t)\right], \quad t \in \mathbb{R},
\end{aligned}
$$

where $\tilde{f}\left(\frac{n \pi}{\sigma}\right)$ and $\tilde{f}^{\prime}-\left(\frac{n \pi}{\sigma}\right)$ are approximate samples of $f\left(\frac{n \pi}{\sigma}\right)$ and $f^{\prime}-\left(\frac{n \pi}{\sigma}\right)$, respectively. Let us assume that the differences $\varepsilon_{n}:=f\left(\frac{n \pi}{\sigma}\right)-\tilde{f}\left(\frac{n \pi}{\sigma}\right), \varepsilon_{n}^{\prime}:=f^{\prime}\left(\frac{n \pi}{\sigma}\right)-\tilde{f}^{\prime}-\left(\frac{n \pi}{\sigma}\right), n \in \mathbb{Z}$, are bounded by a positive number $\varepsilon$, i.e. $\left|\varepsilon_{n}\right|,\left|\varepsilon_{n}^{\prime}\right| \leq \varepsilon$.If $f(t) \in \mathrm{PW}_{\sigma}^{2}$ satisfies the natural decay conditions

$$
\begin{aligned}
& \left|\varepsilon_{n}\right| \leq\left|f\left(\frac{n \pi}{\sigma}\right)\right|, \quad\left|\varepsilon^{\prime}{ }_{n}\right| \leq\left|f^{\prime}\left(\frac{n \pi}{\sigma}\right)\right|, \\
& |f(t)| \leq \frac{M_{f}}{|t|^{\ell+1}}, \quad t \in \mathbb{R}-\{0\},
\end{aligned}
$$

$0<\lambda \leq 1$, then for $0<\varepsilon \leq \min \{\pi / \sigma, \sigma / \pi, 1 / \sqrt{e}\}$, we have, [5],

$$
\|\mathcal{A}(\varepsilon, f)\|_{\infty} \leq \frac{4 e^{1 / 4}}{\sigma(\ell+1)}\left\{\sqrt{3} e(1+\sigma)+\left((\pi / \sigma) A+M_{f}\right) \rho(\varepsilon)+(\sigma+2+\log (2)) M_{f}\right\} \varepsilon \log (1 / \varepsilon),
$$

where

$$
A:=\frac{3 \sigma}{\pi}\left(|f(0)|+M_{f}\left(\frac{\sigma}{\pi}\right)^{\ell}\right), \quad \rho(\varepsilon):=\gamma+10 \log (1 / \varepsilon),
$$

and $\gamma:=\lim _{n \rightarrow \infty}\left[\sum_{k=1}^{n} \frac{1}{k}-\log n\right] \cong 0.577216$ is the Euler-Mascheroni constant. 
The classical [6] sampling theorem of Whittaker, Kotel'nikov and Shannon (WKS) for $f \in \mathrm{PW}_{\sigma}^{2}$ is the series representation

$$
f(t)=\sum_{n=-\infty}^{\infty} f\left(\frac{n \pi}{\sigma}\right) S_{n}(t), \quad t \in \mathbb{R},
$$

where the convergence is absolute and uniform on $\mathbb{R}$ and it is uniform on compact sets of $\mathbb{C}$ (cf. [6-8]). Series (12), which is of Lagrange interpolation type, has been used to compute eigenvalues of second order eigenvalue problems (see e.g. [9-13]). The use of (12) in numerical analysis is known as the sinc-method established by Stenger (cf. [14-16]). In [11,12], the authors applied (12) and the regularized sinc method to compute eigenvalues of Dirac systems with a derivation of the error estimates as given by $[17,18]$. The regularized sinc method; a method which is based on (WKS) but applied to regularized functions. Hence avoiding any (multiple) integration and keeping the number of terms in the Cardinal series manageable. It has been demonstrated that the method is capable of delivering higher order estimates of the eigenvalues at a very low cost. The aim of this article is to investigate the possibilities of using Hermite interpolations rather than Lagrange interpolations, to compute the eigenvalues numerically. Notice that, due to Paley-Wiener's theorem [19] $f \in \mathrm{PW}_{\sigma}^{2}$ if and only if there is $g(\cdot) \in L^{2}$ $(-\sigma, \sigma)$ such that

$$
f(t)=\frac{1}{\sqrt{2 \pi}} \int_{-\sigma}^{\sigma} g(x) e^{i x t} d x .
$$

Therefore $f^{\prime}(t) \in \mathrm{PW}_{\sigma}^{2}$, i.e, $f^{\prime}(t)$ also has an expansion of the form (12). However, $f^{\prime}(t)$ can be also obtained by term-by-term differentiation formula of (12)

$$
f^{\prime}(t)=\sum_{n=-\infty}^{\infty} f\left(\frac{n \pi}{\sigma}\right) S_{n}^{\prime}(t),
$$

see [[6], p. 52] for convergence. Thus the use of Hermite interpolations will not cost any additional computational efforts since the samples $f\left(\frac{n \pi}{\sigma}\right)$ will be used to compute both $f(t)$ and $f^{\prime}(t)$ according to (12) and (14), respectively. We would like to mention that works in direction of computing eigenvalues with the new method, Hermite interpolation technique, are few (see e.g. [5]). Also articles in computing of eigenvalues with discontinuous are few (see [20-22]). However the computing of eigenvalues by Hermite interpolation technique which has discontinuity conditions, do not exist as for as we know. The next section contains some preliminary results. The method with error estimates are contained in Section three. The last section involves some illustrative examples.

\section{The eigenvalue problem}

In this section we closely follow the analysis derived by [23] to establish the needed relations (see also [24]). We consider the Dirac system

$$
\begin{aligned}
& u_{2}^{\prime}(x)-r_{1}(x) u_{1}(x)=\lambda u_{1}(x), \quad u_{1}^{\prime}(x)+r_{2}(x) u_{2}(x)=-\lambda u_{2}(x), \quad x \in[-1,0) \cup(0,1], \\
& U_{1}(u):=\sin \alpha u_{1}(-1)+\cos \alpha u_{2}(-1)=0,
\end{aligned}
$$




$$
U_{2}(u):=\sin \beta u_{1}(1)+\cos \beta u_{2}(1)=0,
$$

and transmission conditions

$$
\begin{aligned}
& U_{3}(u):=u_{1}\left(0^{-}\right)-\delta u_{1}\left(0^{+}\right)=0, \\
& U_{4}(u):=u_{2}\left(0^{-}\right)-\delta u_{2}\left(0^{+}\right)=0,
\end{aligned}
$$

where $\lambda \in \mathbb{C}$; the real valued function $r_{1}(\cdot)$ and $r_{2}(\cdot)$ are continuous in $[-1,0)$ and $(0,1]$, and have finite limits $r_{1}\left(0^{ \pm}\right):=\lim _{x \rightarrow 0^{ \pm}} r_{1}(x), r_{2}\left(0^{ \pm}\right):=\lim _{x \rightarrow 0^{ \pm}} r_{2}(x) ; \delta \in \mathbb{R}, \alpha, \beta \in[0, \pi)$ and $\delta$ $\neq 0$.

Let $\boldsymbol{H}$ be the Hilbert space

$$
H:=\left\{\boldsymbol{u}(x)=\left(\begin{array}{l}
u_{1}(x) \\
u_{2}(x)
\end{array}\right), u_{1}(x), u_{2}(x) \in L^{2}(-1,0) \oplus L^{2}(0,1)\right\} .
$$

The inner product of $\boldsymbol{H}$ is defined by

$$
\langle\boldsymbol{u}(\cdot), \boldsymbol{v}(\cdot)\rangle_{H}:=\int_{-1}^{0} \boldsymbol{u}^{\top}(x) \overline{\boldsymbol{v}}(x) d x+\delta^{2} \int_{0}^{1} \boldsymbol{u}^{\top}(x) \overline{\boldsymbol{v}}(x) d x,
$$

where $T$ denotes the matrix transpose,

$$
\boldsymbol{u}(x)=\left(\begin{array}{l}
u_{1}(x) \\
u_{2}(x)
\end{array}\right), \boldsymbol{v}(x)=\left(\begin{array}{l}
v_{1}(x) \\
v_{2}(x)
\end{array}\right) \in H, \quad u_{i}(\cdot), v_{i}(\cdot) \in L^{2}(-1,1), \quad i=1,2 .
$$

Equation (15) can be written as

$$
\ell(\boldsymbol{u}):=A \boldsymbol{u}^{\prime}(x)-P(x) \boldsymbol{u}(x)=\lambda \boldsymbol{u}(x),
$$

where

$$
A=\left(\begin{array}{ll}
0 & 1 \\
-1 & 0
\end{array}\right), \quad P(x)=\left(\begin{array}{cc}
r_{1}(x) & 0 \\
0 & r_{2}(x)
\end{array}\right), \quad \boldsymbol{u}(x)=\left(\begin{array}{l}
u_{1}(x) \\
u_{2}(x)
\end{array}\right)
$$

For functions $u(x)$, which defined on $[-1,0) \cup(0,1]$ and has finite limit $u( \pm 0):=\lim _{x \rightarrow \pm 0} u(x)$, by $u_{(1)}(x)$ and $u_{(2)}(x)$ we denote the functions

$$
u_{(1)}(x)=\left\{\begin{array}{l}
u(x), \quad x \in[-1,0) ; \\
u\left(0^{-}\right), x=0
\end{array}, u_{(2)}(x)=\left\{\begin{array}{l}
u(x), x \in(0,1] \\
u\left(0^{+}\right), x=0
\end{array}\right.\right.
$$

which are defined on $\Gamma_{1}:=[-1,0]$ and $\Gamma_{2}:=0[1]$ respectively.

In the following lemma, we will prove that the eigenvalues of the problem (15)-(19) are real.

Lemma 2.1 The eigenvalues of the problem (15)-(19) are real.

Proof. Assume the contrary that $\lambda_{0}$ is a nonreal eigenvalue of problem (15)-(19). Let $\left(\begin{array}{l}u_{1}(x) \\ u_{2}(x)\end{array}\right)$ be a corresponding (non-trivial) eigenfunction. By (15), we have, for $x \in[-1,0)$ $\cup(0,1]$,

$$
\frac{d}{d x}\left\{u_{1}(x) \bar{u}_{2}(x)-\bar{u}_{1}(x) u_{2}(x)\right\}=\left(\bar{\lambda}_{0}-\lambda_{0}\right)\left\{\left|u_{1}(x)\right|^{2}+\left|u_{2}(x)\right|^{2}\right\}
$$


Integrating the above equation through $[-1,0)$ and $(0,1]$, we obtain

$$
\begin{aligned}
\left(\bar{\lambda}_{0}-\lambda_{0}\right)\left[\int_{-1}^{0}\left(\left|u_{1}(x)\right|^{2}+\left|u_{2}(x)\right|^{2}\right) d x\right]= & u_{1}\left(0^{-}\right) \bar{u}_{2}\left(0^{-}\right)-\bar{u}_{1}\left(0^{-}\right) u_{2}\left(0^{-}\right) \\
& -\left[u_{1}(-1) \bar{u}_{2}(-1)-\bar{u}_{1}(-1) u_{2}(-1)\right] \\
\left(\bar{\lambda}_{0}-\lambda_{0}\right)\left[\int_{0}^{1}\left(\left|u_{1}(x)\right|^{2}+\left|u_{2}(x)\right|^{2}\right) d x\right]= & u_{1}(1) \bar{u}_{2}(1)-\bar{u}_{1}(1) u_{2}(1) \\
& -\left[u_{1}\left(0^{+}\right) \bar{u}_{2}\left(0^{+}\right)-\bar{u}_{1}\left(0^{+}\right) u_{2}\left(0^{+}\right)\right] .
\end{aligned}
$$

Then from (16), (17) and transmission conditions, we have respectively

$$
\begin{aligned}
& u_{1}(-1) \bar{u}_{2}(-1)-\bar{u}_{1}(-1) u_{2}(-1)=0, \\
& u_{1}(1) \bar{u}_{2}(1)-\bar{u}_{1}(1) u_{2}(1)=0
\end{aligned}
$$

and

$$
u_{1}\left(0^{-}\right) \bar{u}_{2}\left(0^{-}\right)-\bar{u}_{1}\left(0^{-}\right) u_{2}\left(0^{-}\right)=\delta^{2}\left[u_{1}\left(0^{+}\right) \bar{u}_{2}\left(0^{+}\right)-\bar{u}_{1}\left(0^{+}\right) u_{2}\left(0^{+}\right)\right] .
$$

Since $\lambda_{0} \neq \bar{\lambda}_{0}$, it follows from the last three equations and (25), (26) that

$$
\int_{-1}^{0}\left(\left|u_{1}(x)\right|^{2}+\left|u_{2}(x)\right|^{2}\right) d x+\delta^{2} \int_{0}^{1}\left(\left|u_{1}(x)\right|^{2}+\left|u_{2}(x)\right|^{2}\right) d x=0 .
$$

Then $u_{i}(x)=0, i=1,2$ and this is contradiction. Consequently, $\lambda_{0}$ must be real.

Lemma 2.2 Let $\lambda_{1}$ and $\lambda_{2}$ be two different eigenvalues of the problem (15)-(19). Then the corresponding eigenfunctions $\boldsymbol{u}\left(x, \lambda_{1}\right)$ and $\boldsymbol{v}\left(x, \lambda_{2}\right)$ of this problem satisfy the following equality

$$
\int_{-1}^{0} \boldsymbol{u}^{\top}\left(x, \lambda_{1}\right) \boldsymbol{v}\left(x, \lambda_{2}\right) d x+\delta^{2} \int_{0}^{1} \boldsymbol{u}^{\top}\left(x, \lambda_{1}\right) \boldsymbol{v}\left(x, \lambda_{2}\right) d x=0 .
$$

Proof. By (15) we obtain

$$
\frac{d}{d x}\left\{u_{1}\left(x, \lambda_{1}\right) v_{2}\left(x, \lambda_{2}\right)-u_{2}\left(x, \lambda_{2}\right) v_{1}\left(x, \lambda_{1}\right)\right\}=\left(\lambda_{2}-\lambda_{1}\right)\left\{u_{1}\left(x, \lambda_{1}\right) v_{1}\left(x, \lambda_{2}\right)+u_{2}\left(x, \lambda_{1}\right) v_{2}\left(x, \lambda_{2}\right)\right\} .
$$

Integrating the above equation through $[-1,0)$ and $(0,1]$, and taking into account $\boldsymbol{u}$ $\left(x, \lambda_{1}\right)$ and $\boldsymbol{v}\left(x, \lambda_{2}\right)$ satisfy (16)-(19), we obtain (28), where $\lambda_{1} \neq \lambda_{2}$.

Now, we shall construct a special fundamental system of solutions of the Equation (15) for $\lambda$ not being an eigenvalue. Let us consider the next initial value problem:

$$
\begin{aligned}
& u_{2}^{\prime}(x)-r_{1}(x) u_{1}(x)=\lambda u_{1}(x), \quad u_{1}^{\prime}(x)+r_{2}(x) u_{2}(x)=-\lambda u_{2}(x), \quad x \in(-1,0), \\
& u_{1}(-1)=\cos \alpha, \quad u_{2}(-1)=-\sin \alpha .
\end{aligned}
$$

By virtue of Theorem 1.1 in [23] this problem has a unique solution $u_{2}^{\prime}(x)-r_{1}(x) u_{1}(x)=\lambda u_{1}(x), \quad u_{1}^{\prime}(x)+r_{2}(x) u_{2}(x)=-\lambda u_{2}(x), \quad x \in(0,1)$, which $\quad$ is an 
entire function of $\lambda \in \mathbb{C}$ for each fixed $x \in[-1,0]$. Similarly, employing the same method as in proof of Theorem 1.1 in [23], we see that the problem

$$
\begin{aligned}
& u_{2}^{\prime}(x)-r_{1}(x) u_{1}(x)=\lambda u_{1}(x), \quad u_{1}^{\prime}(x)+r_{2}(x) u_{2}(x)=-\lambda u_{2}(x), \quad x \in(0,1), \\
& u_{1}(1)=\cos \beta, \quad u_{2}(1)=-\sin \beta .
\end{aligned}
$$

has a unique solution $\boldsymbol{u}=\left(\begin{array}{l}\chi_{12}(x, \lambda) \\ \chi_{22}(x, \lambda)\end{array}\right)$ which is an entire function of parameter $\lambda$ for each fixed $x \in[0.1]$.

Now the functions $\phi_{i 2}(x, \lambda)$ and $\chi_{i 1}(x, \lambda)$ are defined in terms of $\phi_{i 1}(x, \lambda)$ and $\chi_{i 2}(x$, $\lambda), i=1,2$, respectively, as follows: The initial-value problem,

$$
\begin{aligned}
& u_{2}^{\prime}(x)-r_{1}(x) u_{1}(x)=\lambda u_{1}(x), \quad u_{1}^{\prime}(x)+r_{2}(x) u_{2}(x)=-\lambda u_{2}(x), \quad x \in(0,1), \\
& u_{1}(0)=\frac{1}{\delta} \phi_{11}(0, \lambda), u_{2}(0)=\frac{1}{\delta} \phi_{21}(0, \lambda),
\end{aligned}
$$

has unique solution $\boldsymbol{u}=\left(\begin{array}{l}\phi_{12}(x, \lambda) \\ \phi_{22}(x, \lambda)\end{array}\right)$ for each $\lambda \in \mathbb{C}$.

Similarly, the following problem also has a unique solution $\boldsymbol{u}=\left(\begin{array}{l}\chi_{11}(x, \lambda) \\ \chi_{21}(x, \lambda)\end{array}\right)$ :

$$
\begin{aligned}
& u_{2}^{\prime}(x)-r_{1}(x) u_{1}(x)=\lambda u_{1}(x), \quad u_{1}^{\prime}(x)+r_{2}(x) u_{2}(x)=-\lambda u_{2}(x), \quad x \in(-1,0), \\
& u_{1}(0)=\delta \chi_{12}(0, \lambda), \quad u_{2}(0)=\delta \chi_{22}(0, \lambda)
\end{aligned}
$$

Let us construct two basic solutions of the equation (15) as

$$
\phi(\cdot, \lambda)=\left(\begin{array}{l}
\phi_{1}(\cdot, \lambda) \\
\phi_{2}(\cdot, \lambda)
\end{array}\right), \quad \mathcal{X}(\cdot, \lambda)=\left(\begin{array}{l}
\mathcal{X}_{1}(\cdot, \lambda) \\
\mathcal{X}_{2}(\cdot, \lambda)
\end{array}\right)
$$

where

$$
\begin{aligned}
& \phi_{1}(x, \lambda)=\left\{\begin{array}{l}
\phi_{11}(x, \lambda), x \in[-1,0) \\
\phi_{12}(x, \lambda), x \in(0,1]
\end{array}, \quad \phi_{2}(x, \lambda)=\left\{\begin{array}{l}
\phi_{21}(x, \lambda), x \in[-1,0), \\
\phi_{22}(x, \lambda), x \in(0,1]
\end{array},\right.\right. \\
& \chi_{1}(x, \lambda)=\left\{\begin{array}{l}
\chi_{11}(x, \lambda), x \in[-1,0) \\
\chi_{12}(x, \lambda), x \in(0,1]
\end{array}, \quad \chi_{2}(x, \lambda)=\left\{\begin{array}{l}
\chi_{21}(x, \lambda), x \in[-1,0), \\
\chi_{22}(x, \lambda), x \in(0,1]
\end{array}\right.\right.
\end{aligned}
$$

By virtue of Equations (34) and (36) these solutions satisfy both transmission conditions (18) and (19). These functions are entire in $\lambda$ for all $x \in[-1,0) \cup(0,1]$.

Let $W(\phi, \chi)(\cdot, \lambda)$ denote the Wronskian of $\phi(\cdot, \lambda)$ and $\chi(\cdot, \lambda)$ defined in [[25], p. 194], i.e.,

$$
W(\boldsymbol{\phi}, \chi)(\cdot, \lambda):=\left|\begin{array}{cc}
\phi_{1}(\cdot, \lambda) & \phi_{2}(\cdot, \lambda) \\
\chi_{1}(\cdot, \lambda) & \chi_{2}(\cdot, \lambda)
\end{array}\right|
$$

It is obvious that the Wronskian

$$
\Omega_{i}(\lambda):=W(\boldsymbol{\phi}, \chi)(x, \lambda)=\phi_{1 i}(x, \lambda) \chi_{2 i}(x, \lambda)-\phi_{2 i}(x, \lambda) \chi_{1 i}(x, \lambda), \quad x \in \Gamma_{i,} \quad i=1,2
$$

are independent of $x \in \Gamma_{\mathrm{i}}$ and are entire functions. Taking into account (34) and (36), a short calculation gives 


$$
\Omega_{1}(\lambda)=\delta^{2} \Omega_{2}(\lambda)
$$

for each $\lambda \in \mathbb{C}$.

Corollary 2.3 The zeros of the functions $\Omega_{1}(\lambda)$ and $\Omega_{2}(\lambda)$ coincide.

Then, we may introduce to the consideration the characteristic function $\Omega(\lambda)$ as

$$
\Omega(\lambda):=\Omega_{1}(\lambda)=\delta^{2} \Omega_{2}(\lambda) .
$$

In the following lemma, we show that all eigenvalues of the problem (15)-(19) are simple.

Lemma 2.4 All eigenvalues of problem (15)-(19) are just zeros of the function $\Omega(\lambda)$. Moreover, every zero of $\Omega(\lambda)$ has multiplicity one.

Proof. Since the functions $\phi_{1}(x, \lambda)$ and $\phi_{2}(x, \lambda)$ satisfy the boundary condition (16) and both transmission conditions (18) and (19), to find the eigenvalues of the (15)-(19) we have to insert the functions $\phi_{1}(x, \lambda)$ and $\phi_{2}(x, \lambda)$ in the boundary condition (17) and find the roots of this equation.

By (15) we obtain for $\lambda, \mu \in \mathbb{C}, \lambda \neq \mu$,

$$
\frac{d}{d x}\left\{\phi_{1}(x, \lambda) \phi_{2}(x, \mu)-\phi_{1}(x, \mu) \phi_{2}(x, \lambda)\right\}=(\mu-\lambda)\left\{\phi_{1}(x, \lambda) \phi_{1}(x, \mu)+\phi_{2}(x, \lambda) \phi_{2}(x, \mu)\right\} .
$$

Integrating the above equation through $[-1,0)$ and $(0,1]$, and taking into account the initial conditions (30), (34) and (36), we obtain

$$
\begin{aligned}
& \phi_{12}(1, \lambda) \phi_{22}(1, \mu)-\phi_{12}(1, \mu) \phi_{22}(1, \lambda)= \\
&(\mu-\lambda)\left(\int_{-1}^{0}\left(\phi_{11}(x, \lambda) \phi_{11}(x, \mu)+\phi_{21}(x, \lambda) \phi_{21}(x, \mu)\right) d x\right. \\
&\left.+\delta^{2} \int_{0}^{1}\left(\phi_{12}(x, \lambda) \phi_{12}(x, \mu)+\phi_{22}(x, \lambda) \phi_{22}(x, \mu)\right) d x\right) .
\end{aligned}
$$

Dividing both sides of (41) by $(\lambda-\mu)$ and by letting $\mu \rightarrow \lambda$, we arrive to the relation

$$
\begin{aligned}
\phi_{22}(1, \lambda) \frac{\partial \phi_{12}(1, \lambda)}{\partial \lambda}-\phi_{12}(1, \lambda) \frac{\partial \phi_{22}(1, \lambda)}{\partial \lambda}= & -\left(\int_{-1}^{0}\left(\left|\phi_{11}(x, \lambda)\right|^{2}+\left|\phi_{21}(x, \lambda)\right|^{2}\right) d x\right. \\
& \left.+\delta^{2} \int_{0}^{1}\left(\left|\phi_{12}(x, \lambda)\right|^{2}+\left|\phi_{22}(x, \lambda)\right|^{2}\right) d x\right) .
\end{aligned}
$$

We show that equation

$$
\Omega(\lambda)=-\delta^{2}\left(\sin \beta \phi_{12}(1, \lambda)+\cos \beta \phi_{22}(1, \lambda)\right)=0
$$

has only simple roots. Assume the converse, i.e., Equation (43) has a double root $\lambda^{*}$, say. Then the following two equations hold

$$
\begin{aligned}
& \sin \beta \phi_{12}\left(1, \lambda^{*}\right)+\cos \beta \phi_{22}\left(1, \lambda^{*}\right)=0, \\
& \sin \beta \frac{\partial \phi_{12}\left(1, \lambda^{*}\right)}{\partial \lambda}+\cos \beta \frac{\partial \phi_{22}\left(1, \lambda^{*}\right)}{\partial \lambda}=0 .
\end{aligned}
$$


The Equations (44) and (45) imply that

$$
\phi_{22}\left(1, \lambda^{*}\right) \frac{\partial \phi_{12}\left(1, \lambda^{*}\right)}{\partial \lambda}-\phi_{12}\left(1, \lambda^{*}\right) \frac{\partial \phi_{22}\left(1, \lambda^{*}\right)}{\partial \lambda}
$$

Combining (46) and (42), with $\lambda=\lambda^{*}$, we obtain

$$
\int_{-1}^{0}\left(\left|\phi_{11}\left(x,, \lambda^{*}\right)\right|^{2}+\left|\phi_{21}\left(x, \lambda^{*}\right)\right|^{2}\right) d x+\delta^{2} \int_{0}^{1}\left(\left|\phi_{12}\left(x, \lambda^{*}\right)\right|^{2}+\left|\phi_{22}\left(x, \lambda^{*}\right)\right|^{2}\right) d x=0 .
$$

It follows that $\phi_{1}\left(x, \lambda^{*}\right)=\phi_{2}\left(x, \lambda^{*}\right)=0$, which is impossible. This proves the lemma.

Here $\left\{\boldsymbol{\phi}\left(\cdot, \lambda_{n}\right)\right\}_{n=-\infty}^{\infty}$ will be a sequence of eigen-vector-functions of (15)-(19) corresponding to the eigenvalues $\left\{\lambda_{n}\right\}_{n=-\infty}^{\infty}$.Since $\chi(\cdot, \lambda)$ satisfies (17)-(19), then the eigenvalues are also determined via

$$
\sin \alpha \chi_{11}(-1, \lambda)+\cos \alpha \chi_{21}(-1, \lambda)=\Omega(\lambda) .
$$

Therefore $\left\{\chi\left(\cdot, \lambda_{n}\right)\right\}_{n=-\infty}^{\infty}$ is another set of eigen-vector-functions which is related by $\left\{\boldsymbol{\phi}\left(\cdot, \lambda_{n}\right)\right\}_{n=-\infty}^{\infty}$ with

$$
\chi\left(x, \lambda_{n}\right)=c_{n} \phi\left(x, \lambda_{n}\right), \quad x \in[-1,0) \cup(0,1], \quad n \in \mathbb{Z},
$$

Where $c_{n} \neq 0$ are non-zero constants, since all eigenvalues are simple. Since the eigenvalues are all real, we can take the eigen-vector-functions to be real valued.

Since $\phi(\cdot, \lambda)$ satisfies (16), then the eigenvalues of the problem (15)-(19) are the zeros of the function

$$
\Omega(\lambda)=-\delta^{2}\left(\sin \beta \phi_{12}(1, \lambda)+\cos \beta \phi_{22}(1, \lambda)\right) .
$$

Notice that both $\phi(\cdot, \lambda)$ and $\Omega(\lambda)$ are entire functions of $\lambda$. Now we shall transform Equations (15), (30), (34) and (37) into the integral equations (see [25]),

$$
\begin{aligned}
& \phi_{11}(x, \lambda)=\cos (\lambda(x+1)-\alpha)-\mathcal{S}_{-1,1} \phi_{11}(x, \lambda)-\tilde{\mathcal{S}}_{-1,2} \phi_{21}-(x, \lambda), \\
& \phi_{21}(x, \lambda)=\sin (\lambda(x+1)-\alpha)+\tilde{\mathcal{S}}_{-1,1} \phi_{11}(x, \lambda)-\mathcal{S}_{-1,2} \phi_{21}(x, \lambda), \\
& \phi_{12}(x, \lambda)=\frac{1}{\delta} \phi_{11}\left(0^{-}, \lambda\right) \cos (\lambda x)-\frac{1}{\delta} \phi_{21}\left(0^{-}, \lambda\right) \sin (\lambda x)-\mathcal{S}_{0,1} \phi_{12}(x, \lambda)-\tilde{\mathcal{S}}_{0,2} \phi_{22}(x, \lambda), \\
& \phi_{22}(x, \lambda)=\frac{1}{\delta} \phi_{11}\left(0^{-}, \lambda\right) \sin (\lambda x)+\frac{1}{\delta} \phi_{21}\left(0^{-}, \lambda\right) \cos (\lambda x)^{-}+\tilde{\mathcal{S}}_{0,1} \phi_{12}(x, \lambda)-\mathcal{S}_{0,2} \phi_{22}(x, \lambda),
\end{aligned}
$$

where $\mathcal{S}_{-1, i}, \tilde{\mathcal{S}}_{-1, i}, \mathcal{S}_{0, i}$ and $\tilde{\mathcal{S}}_{0, i}, i=1,2$, are the Volterra integral operators defined by

$$
\begin{aligned}
& \mathcal{S}_{-1, i} \varphi(x, \lambda):=\int_{-1}^{x} \sin \lambda(x-t) r_{i}(t) \varphi(t, \lambda) d t, \tilde{\mathcal{S}}_{-1, i} \varphi(x, \lambda):=\int_{-1}^{x} \cos \lambda(x-t) r_{i}(t) \varphi(t, \lambda) d t, \\
& \mathcal{S}_{0, i} \varphi(x, \lambda):=\int_{0}^{x} \sin \lambda(x-t) r_{i}(t) \varphi(t, \lambda) d t, \quad \tilde{\mathcal{S}}_{0, i} \varphi(x, \lambda):=\int_{0}^{x} \cos \lambda(x-t) r_{i}(t) \varphi(t, \lambda) d t .
\end{aligned}
$$


For convenience, we define the constants

$$
\begin{aligned}
& c_{1}:=\int_{-1}^{0}\left[\left|r_{1}(t)\right|+\left|r_{2}(t)\right|\right] d t, \quad c_{2}:=c_{1} \exp \left(c_{1}\right), \\
& c_{3}:=\int_{0}^{1}\left[\left|r_{1}(t)\right|+\left|r_{2}(t)\right|\right] d t, \quad c_{4}:=c_{2}+\frac{2}{|\delta|}\left(1+c_{2}\right) .
\end{aligned}
$$

Define $h_{-1, i}(\cdot, \lambda)$ and $h_{0, i}(\cdot, \lambda), i=1,2$, to be

$$
\left.\begin{array}{l}
\left.\begin{array}{l}
h_{-1,1}(x, \lambda):=\mathcal{S}_{-1,1} \phi_{11}(x, \lambda)+\tilde{\mathcal{S}}_{-1,2} \phi_{21}(x, \lambda), \\
h_{-1,2}(x, \lambda):=\tilde{\mathcal{S}}_{-1,1} \phi_{11}(x, \lambda)+\mathcal{S}_{-1,2} \phi_{21}(x, \lambda),
\end{array}\right\} \\
h_{0,1}(x, \lambda):=\mathcal{S}_{0,1} \phi_{12}(x, \lambda)+\tilde{\mathcal{S}}_{0,2} \phi_{22}(x, \lambda), \\
h_{0,2}(x, \lambda):=\tilde{\mathcal{S}}_{0,1} \phi_{12}(x, \lambda)-\mathcal{S}_{0,2} \phi_{22}(x, \lambda) .
\end{array}\right\}
$$

Lemma 2.5 The functions $h_{-1,1}(x, \lambda)$ and $h_{-1,2}(x, \lambda)$ are entire in $\lambda$ for any fixed $x \in$ $[-1,0)$ and satisfy the growth condition

$$
\left|h_{-1,1}(x, \lambda)\right|,\left|h_{-1,2}(x, \lambda)\right| \leq 2 c_{2} e^{|\Im \lambda|(x+1)}, \quad \lambda \in \mathbb{C} .
$$

Proof. Since $h_{-1,1}(x, \lambda)=\mathcal{S}_{-1,1} \phi_{11}(x, \lambda)+\tilde{\mathcal{S}}_{-1,2} \phi_{21}(x, \lambda)$, then from (51) and (52) we obtain $h_{-1,1}(x, \lambda)=\mathcal{S}_{-1,1} \cos (\lambda(x+1)-\alpha)+\tilde{\mathcal{S}}_{-1,2} \sin (\lambda(x+1)-\alpha)-\mathcal{S}_{-1,1} h_{-1,1}-(x, \lambda)+\tilde{\mathcal{S}}_{-1,2} h_{-1,2}(x, \lambda)$ Using the inequalities $|\sin z| \leq e^{|\Im z z|}$ and $|\cos z| \leq e^{|\Im z|}$ for $z \in \mathbb{C}$, leads for $\lambda \in \mathbb{C}$ to

$$
\begin{aligned}
\left|h_{-1,1}(x, \lambda)\right| \leq & \left|\mathcal{S}_{-1,1} \cos (\lambda(x+1)-\alpha)\right|+\left|\tilde{\mathcal{S}}_{-1,2} \sin (\lambda(x-+1)-\alpha)\right|+\left|\mathcal{S}_{-1,1} h_{-1,1}(x, \lambda)\right| \\
& +\left|\tilde{\mathcal{S}}_{-1,2} h_{-1,2}(x, \lambda)\right| \\
\leq & e^{|\Im \lambda|(x+1)} \int_{-1}^{x}\left[\left|r_{1}(t)\right|\left|h_{-1,1}(t, \lambda)\right|+\left|r_{2}(t)\right|\left|h_{-1,2}(t, \lambda)\right|\right] e^{-|\Im \lambda| \mid(t+1)} d t \\
& +2 e^{|\Im \lambda|(x+1)} \int_{-1}^{x}\left[\left|r_{1}(t)\right|+\left|r_{2}(t)\right|\right] d t \\
\leq & 2 c_{1} e^{|\Im \lambda|(x+1)}+e^{|\Im \lambda| \mid(x+1)} \int_{-1}^{x}\left[\left|r_{1}(t)\right|\left|h_{-1,1}(t, \lambda)\right|+\left|r_{2}(t)\right|\left|h_{-1,2}(t, \lambda)\right|\right] e^{-|\Im \lambda|(t+1)} d t .
\end{aligned}
$$

The above inequality can be reduced to

$$
e^{-|\xi \lambda|(x+1) \mid}\left|h_{-1,1}(x, \lambda)\right| \leq 2 c_{1}+\int_{-1}^{x}\left[\left|r_{1}(t)\right|\left|h_{-1,1}(t, \lambda)\right|+\left|r_{2}(t)\right|\left|h_{-1,2}(t, \lambda)\right|\right] e^{-|\xi \lambda|(t+1)} d t .
$$

Similarly, we can prove that

$$
e^{-|\xi \lambda|(x+1) \mid}\left|h_{-1,2}(x, \lambda)\right| \leq 2 c_{1}+\int_{-1}^{x}\left[\left|r_{1}(t)\right|\left|h_{-1,1}(t, \lambda)\right|+\left|r_{2}(t)\right|\left|h_{-1,2}(t, \lambda)\right|\right] e^{-|\xi \lambda|(t+1)} d t .
$$

Then from (58) and (59) and and Lemma 3.1 of [[25], pp. 204], we obtain (58).

In a similar manner, we will prove the following lemma for $h_{0,1}(\cdot, \lambda)$ and $h_{0,2}(\cdot, \lambda)$.

Lemma 2.6 The functions $h_{0,1}(x, \lambda)$ and $h_{0,2}(x, \lambda)$ are entire in $\lambda$ for any fixed $x \in(0$, 1] and satisfy the growth condition 


$$
\left|h_{0,1}(x, \lambda)\right|,\left|h_{0,2}(x, \lambda)\right| \leq 2 c_{3} c_{4} e^{|\Im \lambda|(x+1)}, \quad \lambda \in \mathbb{C} .
$$

Proof. Since $h_{0,1}(x, \lambda)=\mathcal{S}_{0,1} \phi_{11}(x, \lambda)+\tilde{\mathcal{S}}_{0,2} \phi_{21}(x, \lambda)$, then from (53) and (54) we obtain

$$
\begin{aligned}
h_{0,1}(x, \lambda)= & \frac{1}{\delta} \phi_{11}\left(0^{-}, \lambda\right) \mathcal{S}_{0,1} \cos (\lambda x)-\frac{1}{\delta} \phi_{21}\left(0^{-}, \lambda\right) \tilde{\mathcal{S}}_{0,1} \sin (\lambda x)-\mathcal{S}_{0,1} h_{-1,2}(x, \lambda) \\
& +\frac{1}{\delta} \phi_{11}\left(0^{-}, \lambda\right) \tilde{\mathcal{S}}_{0,2} \sin \left(\lambda_{X}\right)+\frac{1}{\delta} \phi_{21}\left(0^{-}, \lambda\right) \tilde{\mathcal{S}}_{0,2} \cos \left(\lambda_{X}\right)+\tilde{\mathcal{S}}_{0,2} h_{-1,2}(x, \lambda) .
\end{aligned}
$$

Then from (51) and (52) and Lemma 2.5, we get

$$
\begin{aligned}
h_{0,1}(x, \lambda) \leq & \frac{1}{|\delta|}\left|\phi_{11}\left(0^{-}, \lambda\right)\right|\left|\mathcal{S}_{0,1} \cos (\lambda x)\right|+\frac{1}{|\delta|}\left|\phi_{21}\left(0^{-}, \lambda\right)\right|\left|\mathcal{S}_{0,1} \sin (\lambda x)\right| \\
& +\left|\mathcal{S}_{0,1} h_{-1,2}(x, \lambda)\right|+\frac{1}{|\delta|}\left|\phi_{11}\left(0^{-}, \lambda\right)\right|\left|\tilde{\mathcal{S}}_{0,2} \sin (\lambda x)\right| \\
& +\frac{1}{|\delta|}\left|\phi_{21}\left(0^{-}, \lambda\right)\right|\left|\tilde{\mathcal{S}}_{0,2} \cos (\lambda x)\right|-+\left|\tilde{\mathcal{S}}_{0,2} h_{-1,2}(x, \lambda)\right| \\
\leq & 2\left(c_{2}+\frac{2}{|\delta|}\left(1+c_{2}\right)\right) c_{3} e^{|\Im \lambda|(x+1)}=2 c_{3} c_{4} e^{|\Im \lambda|(x+1)} .
\end{aligned}
$$

Similarly, we can prove that

$$
h_{0,2}(x, \lambda) \leq 2 c_{3} c_{4} e^{|\Im \lambda|(x+1)} .
$$

\section{The numerical scheme}

In this section we derive the method of computing eigenvalues of problem (15)-(19) numerically. The basic idea of the scheme is to split $\Omega(\lambda)$ into two parts a known part $\mathcal{K}(\lambda)$ and an unknown one $\mathcal{U}(\lambda)$. Then we prove that $\mathcal{U}(\lambda)$ has an expansion of the form (1). We then approximate $\mathcal{U}(\lambda)$ in two stages. First by truncating the sampling expansion (4) and then by approximating the samples, using standard methods of solving ordinary differential equations. This produces both a truncation error and an amplitude error. We apply forms (4) and (7) to derive an estimate of the error of the technique. We first split $\Omega(\lambda)$ into two parts:

$$
\Omega(\lambda):=\mathcal{K}(\lambda)+\mathcal{U}(\lambda)
$$

where $\mathcal{U}(\lambda)$ is the unknown part involving integral operators

$$
\mathcal{U}(\lambda):=\delta^{2} \sin \beta h_{0,1}(1, \lambda)-\delta^{2} \cos \beta h_{0,2}(1, \lambda)-\delta \sin (\lambda-\beta) h_{-1,1}\left(0^{-}, \lambda\right)-\delta \cos (\lambda+\beta) h_{-1,2}\left(0^{-}, \lambda\right),
$$

and $\mathcal{K}(\lambda)$ is the known part

$$
\mathcal{K}(\lambda):=-\delta \sin (2 \lambda-\alpha+\beta) .
$$

Then, from Lemmas 2.5 and 2.6, we have the following result.

Lemma 3.1 The function $\mathcal{U}(\lambda)$ is entire in $\lambda$ and the following estimate holds

$$
|\mathcal{U}(\lambda)| \leq M e^{2|\Im \lambda|}
$$

where

$$
M:=4|\delta|\left(c_{2}+|\delta| c_{3} c_{4}\right) .
$$


Proof. From (63), we have

$$
\begin{aligned}
|\mathcal{U}(\lambda)| \leq & |\delta|^{2}|\sin \beta|\left|h_{0,1}(1, \lambda)\right|+|\delta|^{2}|\cos \beta|\left|h_{0,2}(1, \lambda)\right| \\
& +|\delta||\sin (\lambda-\beta)|\left|h_{-1,1}\left(0^{-}, \lambda\right)\right|+|\delta||\cos (\lambda+\beta)|\left|h_{-1,2}\left(0^{-}, \lambda\right)\right| .
\end{aligned}
$$

Using the inequalities $|\sin \lambda| \leq e^{|\Im \lambda|}$ and $|\cos \lambda| \leq e^{|\Im \lambda|}$ for $\lambda \in \mathbb{C}$, and Lemmas 2.5 and 2.6 imply (65).

Let $\theta \in(0,1)$ and $m \in \mathbb{Z}^{+}, m>1$ be fixed. Let $\mathcal{G}_{\theta, m}(\lambda)$ be the function

$$
\mathcal{G}_{\theta, m}(\lambda):=\left(\frac{\sin \theta \lambda}{\theta \lambda}\right)^{m} \mathcal{U}(\lambda), \quad \lambda \in \mathbb{C} .
$$

Lemma 3.2 $\mathcal{G}_{\theta, m}(\lambda)$ is an entire function of $\lambda$ which satisfies the estimate

$$
\left|\mathcal{G}_{\theta, m}(\lambda)\right| \leq \frac{c_{0}^{m} M}{(1+\theta|\lambda|)^{m}} e^{|\Im \lambda|(2+m \theta)}, \quad \lambda \in \mathbb{C} .
$$

Moreover, $\lambda^{m-1} \mathcal{G}_{\theta, m}(\lambda) \in L^{2}(\mathbb{R})$ and

$$
E_{m-1}\left(\mathcal{G}_{\theta, m}\right)=\sqrt{\int_{-\infty}^{\infty}\left|\lambda^{m-1} \mathcal{G}_{\theta m}(\lambda)\right|^{2} d \lambda} \leq \sqrt{2} c_{0}^{m} M \xi_{0}
$$

where

$$
\xi_{0}:=\sqrt{\frac{1}{\theta^{2 m-1}(2 m-1)}} .
$$

Proof. Since $\mathcal{U}(\lambda)$ is entire, then also $\mathcal{G}_{\theta, m}(\lambda)$ is entire in $\lambda$. Combining the estimates $\left|\frac{\sin z}{z}\right| \leq \frac{c_{0}}{1+|z|} e^{|\Im z|}$, where $c_{0} \simeq 1.72$, cf. [26], and (65), we obtain

$$
\begin{aligned}
\left|\lambda^{m-1} \mathcal{G}_{\theta, m}(\lambda)\right| & \leq\left(\frac{c_{0}}{1+\theta|\lambda|}\right)^{m} e^{|\Im z| m \theta} \cdot M|\lambda|^{m-1} e^{2|\Im \lambda|} \\
& =\frac{c_{0}^{m} M|\lambda|^{m-1}}{(1+\theta|\lambda|)^{m}} e^{|\Im \lambda|(2+m \theta)}, \quad \lambda \in \mathbb{C} .
\end{aligned}
$$

Therefore if $\lambda \in \mathbb{R}$ we have

$$
\left|\lambda^{m-1} \mathcal{G}_{\theta, m}(\lambda)\right| \leq \frac{c_{0}^{m} M|\lambda|^{m-1}}{(1+\theta|\lambda|)^{m}}, \quad \lambda \in \mathbb{R},
$$

i.e. $\lambda^{m-1} \mathcal{G}_{\theta, m}(\lambda) \in L^{2}(\mathbb{R})$. Moreover

$$
\int_{-\infty}^{\infty}\left|\lambda^{m-1} \mathcal{G}_{\theta, m}(\lambda)\right|^{2} d \lambda \leq c_{0}^{2 m} M^{2} \int_{-\infty}^{\infty} \frac{|\lambda|^{2 m-2}}{(1+\theta|\lambda|)^{2 m}} d \lambda=2 c_{0}^{2 m} M^{2} \xi_{0}^{2} .
$$

A direct and important result of Lemma 51 is that $\mathcal{G}_{\theta, m}(\lambda)$ belongs to the Paley-Wienerz space $\mathrm{PW}_{\sigma}^{2}$ with $\sigma=1+m \theta$. Since $\mathcal{G}_{\theta, m}(\lambda) \in \mathrm{PW}_{\sigma}^{2} \subset \mathrm{PW}_{2 \sigma}^{2}$, then we can reconstruct the functions $\mathcal{G}_{\theta, m}(\lambda)$ via the following sampling formula

$$
\mathcal{G}_{\theta, m}(\lambda)=\sum_{n=-\infty}^{\infty}\left[\mathcal{G}_{\theta, m}\left(\frac{n \pi}{\sigma}\right) S_{n}^{2}(\lambda)+\mathcal{G}_{\theta, m}^{\prime}\left(\frac{n \pi}{\sigma}\right) \frac{\sin (\sigma \lambda-n \pi)}{\sigma} S_{n}(\lambda)\right] .
$$


Let $N \in \mathbb{Z}^{+}, N>m$ and approximate $\mathcal{G}_{\theta, m}(\lambda)$ by its truncated series $\mathcal{G}_{\theta, m, N}(\lambda)$, where

$$
\mathcal{G}_{\theta, m, N}(\lambda):=\sum_{n=-N}^{N}\left[\mathcal{G}_{\theta, m}\left(\frac{n \pi}{\sigma}\right) S_{n}^{2}(\lambda)+\mathcal{G}_{\theta, m}^{\prime}\left(\frac{n \pi}{\sigma}\right) \frac{\sin (\sigma \lambda-n \pi)}{\sigma} S_{n}(\lambda)\right] .
$$

Since all eigenvalues are real, then from now on we restrict ourselves to $\lambda \in \mathbb{R}$. Since $\lambda^{m-1} \mathcal{G}_{\theta, m}(\lambda) \in L^{2}(\mathbb{R})$, the truncation error, cf. (5), is given for

$$
\left|\mathcal{G}_{\theta, m}(\lambda)-\mathcal{G}_{\theta, m, N}(\lambda)\right| \leq T_{N, m-1, \sigma}(\lambda),
$$

where

$$
\begin{aligned}
T_{N, m-1, \sigma}(\lambda):= & \frac{\xi_{m-1, \sigma} E_{m-1}|\sin \sigma \lambda|^{2}}{\sqrt{3}(N+1)^{m-1}}\left(\frac{1}{(N \pi-\sigma \lambda)^{3 / 2}}+\frac{1}{(N \pi+\sigma \lambda)^{3 / 2}}\right) \\
& +\frac{\xi_{m-1, \sigma}\left(\sigma E_{m-1}+(m-1) E_{m-2}\right)|\sin \sigma \lambda|^{2}}{\sigma(N+1)^{m-1}}\left(\frac{1}{\sqrt{N \pi-\sigma \lambda}}+\frac{1}{\sqrt{N \pi+\sigma \lambda}}\right) .
\end{aligned}
$$

The samples $\left\{\mathcal{G}_{\theta, m}\left(\frac{n \pi}{\sigma}\right)\right\}_{n=-N}^{N}$ and $\left\{\mathcal{G}^{\prime}{ }_{\theta, m}\left(\frac{n \pi}{\sigma}\right)\right\}_{n=-N}^{N}$, in general, are not known explicitly. So we approximate them by solving numerically $2 N+1$ initial value problems at the nodes $\left\{\frac{n \pi}{\sigma}\right\}_{n=-N}^{N}$. Let $\left\{\tilde{\mathcal{G}}_{\theta, m}\left(\frac{n \pi}{\sigma}\right)\right\}_{n=-N}^{N}$ and $\left\{\tilde{\mathcal{G}}_{\theta, m}^{\prime}\left(\frac{n \pi}{\sigma}\right)\right\}_{n=-N}^{N}$ be the approximations of the samples of $\left\{\mathcal{G}_{\theta, m}\left(\frac{n \pi}{\sigma}\right)\right\}_{n=-N}^{N}$ and $\left\{\mathcal{G}_{\theta, m}^{\prime}\left(\frac{n \pi}{\sigma}\right)\right\}_{n=-N}^{N}$, respectively. Now we define $\tilde{\mathcal{G}}_{\theta, m, N}(\lambda)$, which approximates $\mathcal{G}_{\theta, m, N}(\lambda)$

$$
\tilde{\mathcal{G}}_{\theta, m, N}(\lambda):=\sum_{n=-N}^{N}\left[\tilde{\mathcal{G}}_{\theta, m}\left(\frac{n \pi}{\sigma}\right) S_{n}^{2}(\lambda)+\tilde{\mathcal{G}}_{\theta, m}^{\prime}\left(\frac{n \pi}{\sigma}\right) \frac{\sin (\sigma \lambda-n \pi)}{\sigma} S_{n}(\lambda)\right], \quad N>m .
$$

Using standard methods for solving initial problems, we may assume that for $|n|<N$,

$$
\left|\mathcal{G}_{\theta, m}\left(\frac{n \pi}{\sigma}\right)-\tilde{\mathcal{G}}_{\theta, m}\left(\frac{n \pi}{\sigma}\right)\right|<\varepsilon, \quad\left|\mathcal{G}_{\theta, m}^{\prime}\left(\frac{n \pi}{\sigma}\right)-\tilde{\mathcal{G}}_{\theta, m}^{\prime}\left(\frac{n \pi}{\sigma}\right)\right|<\varepsilon,
$$

for a sufficiently small $\varepsilon$. From Lemma 3.2 we can see that $\mathcal{G}_{\theta, m}(\lambda)$ satisfies the condition (9) when $m>1$ and therefore whenever $0<\varepsilon \leq \min \{\pi / \sigma, \sigma / \pi, 1 / \sqrt{e}\}$ we have

$$
\left|\mathcal{G}_{\theta, m, N}(\lambda)-\tilde{\mathcal{G}}_{\theta, m, N}(\lambda)\right| \leq \mathcal{A}(\varepsilon), \quad \lambda \in \mathbb{R},
$$

where there is a positive constant $M_{\mathcal{G}_{\theta, m}}$ for which, cf. (10),

$$
\mathcal{A}(\varepsilon):=\frac{2 e^{1 / 4}}{\sigma}\left\{\sqrt{3} e(1+\sigma)+\left(\frac{\pi}{\sigma} A+M_{\mathcal{G}_{\theta, m}}\right) \rho(\varepsilon)+(\sigma+2+\log (2)) M_{\mathcal{G}_{\theta, m}}\right\} \varepsilon \log (1 / \varepsilon) .
$$

Here

$$
A:=\frac{3 \sigma}{\pi}\left(\left|\mathcal{G}_{\theta, m}(0)\right|+\frac{\sigma}{\pi} M_{\mathcal{G}_{\theta, m}}\right), \quad \rho(\varepsilon):=\gamma+10 \log (1 / \varepsilon), \quad \ell=1 .
$$

In the following we use the technique of [27] to determine enclosure intervals for the eigenvalues. Let $\lambda^{\prime \prime}$ be an eigenvalue, that is

$$
\Omega\left(\lambda^{*}\right)=\mathcal{K}\left(\lambda^{*}\right)+\left(\frac{\sin \theta \lambda^{*}}{\theta \lambda^{*}}\right)^{-m} \mathcal{G}_{\theta, m}\left(\lambda^{*}\right)=0 .
$$


Then it follows that

$$
\begin{aligned}
\mathcal{K}\left(\lambda^{*}\right)+\left(\frac{\sin \theta \lambda^{*}}{\theta \lambda^{*}}\right)^{-m} \tilde{\mathcal{G}}_{\theta, m, N}\left(\lambda^{*}\right)= & \left(\frac{\sin \theta \lambda^{*}}{\theta \lambda^{*}}\right)^{-m} \tilde{\mathcal{G}}_{\theta, m, N}\left(\lambda^{*}\right)-\left(\frac{\sin \theta \lambda^{*}}{\theta \lambda^{*}}\right)^{-m} \mathcal{G}_{\theta, m}\left(\lambda^{*}\right) \\
= & {\left[\left(\frac{\sin \theta \lambda^{*}}{\theta \lambda^{*}}\right)^{-m} \tilde{\mathcal{G}}_{\theta, m, N}\left(\lambda^{*}\right)-\left(\frac{\sin \theta \lambda^{*}}{\theta \lambda^{*}}\right)^{-m} \mathcal{G}_{\theta, m, N}\left(\lambda^{*}\right)\right] } \\
& +\left[\left(\frac{\sin \theta \lambda^{*}}{\theta \lambda^{*}}\right)^{-m} \mathcal{G}_{\theta, m, N}\left(\lambda^{*}\right)-\left(\frac{\sin \theta \lambda^{*}}{\theta \lambda^{*}}\right)^{-m} \mathcal{G}_{\theta, m}\left(\lambda^{*}\right)\right]
\end{aligned}
$$

and so

$$
\left|\mathcal{K}\left(\lambda^{*}\right)+\left(\frac{\sin \theta \lambda^{*}}{\theta \lambda^{*}}\right)^{-m} \tilde{\mathcal{G}}_{\theta, m, N}\left(\lambda^{*}\right)\right| \leq\left|\frac{\sin \theta \lambda^{*}}{\theta \lambda^{*}}\right|^{-m}\left(T_{N, m-1, \sigma}\left(\lambda^{*}\right)+\mathcal{A}(\varepsilon)\right) .
$$

Since $\mathcal{K}\left(\lambda^{*}\right)+\left(\frac{\sin \theta \lambda^{*}}{\theta \lambda^{*}}\right)^{-m} \tilde{\mathcal{G}}_{\theta, m, N}\left(\lambda^{*}\right)$ is given and, $\left|\frac{\sin \theta \lambda^{*}}{\theta \lambda^{*}}\right|^{-m}\left(T_{N, m-1, \sigma}\left(\lambda^{*}\right)+\mathcal{A}(\varepsilon)\right)$ has computable upper bound, we can define an enclosure for $\lambda^{\prime \prime}$, by solving the following system of inequalities

$$
\begin{aligned}
-\left|\frac{\sin \theta \lambda^{*}}{\theta \lambda^{*}}\right|^{-m} & \left(T_{N, m-1, \sigma}\left(\lambda^{*}\right)+\mathcal{A}(\varepsilon)\right) \leq \mathcal{K}\left(\lambda^{*}\right) \\
& +\left(\frac{\sin \theta \lambda^{*}}{\theta \lambda^{*}}\right)^{-m} \tilde{\mathcal{G}}_{\theta, m, N}\left(\lambda^{*}\right) \leq\left|\frac{\sin \theta \lambda^{*}}{\theta \lambda^{*}}\right|^{-m}\left(T_{N, m-1, \sigma}\left(\lambda^{*}\right)+\mathcal{A}(\varepsilon)\right) .
\end{aligned}
$$

Its solution is an interval containing $\lambda^{*}$, and over which the graph $\mathcal{K}\left(\lambda^{*}\right)+\left(\frac{\sin \theta \lambda^{*}}{\theta \lambda^{*}}\right)^{-m} \times \tilde{\mathcal{G}}_{\theta, m, N}\left(\lambda^{*}\right)$ is squeezed between the graphs

$$
-\left|\frac{\sin \theta \lambda^{*}}{\theta \lambda^{*}}\right|^{-m}\left(T_{N, m-1, \sigma}\left(\lambda^{*}\right)+\mathcal{A}(\varepsilon)\right)
$$

and

$$
\left|\frac{\sin \theta \lambda^{*}}{\theta \lambda^{*}}\right|^{-m}\left(T_{N, m-1, \sigma}\left(\lambda^{*}\right)+\mathcal{A}(\varepsilon)\right) .
$$

Using the fact that

$$
\tilde{\mathcal{G}}_{\theta, m, N}(\lambda) \rightarrow \mathcal{G}_{\theta, m}(\lambda)
$$

uniformly over any compact set, and since $\lambda^{*}$ is a simple root, we obtain for large $N$ and sufficiently small $\varepsilon$

$$
\frac{\partial}{\partial \lambda}\left(\mathcal{K}(\lambda)+\left(\frac{\sin \theta \lambda}{\theta \lambda}\right)^{-m} \tilde{\mathcal{G}}_{\theta, m, N}(\lambda)\right) \neq 0
$$

in a neighborhood of $\lambda^{*}$. Hence the graph of $\mathcal{K}(\lambda)+\left(\frac{\sin \theta \lambda}{\theta \lambda}\right)^{-m} \tilde{\mathcal{G}}_{\theta, m, N}(\lambda)$ intersects the graphs $-\left|\frac{\sin \theta \lambda}{\theta \lambda}\right|^{-m}\left(T_{N, m-1, \sigma}(\lambda)+\mathcal{A}(\varepsilon)\right)$ and $\left|\frac{\sin \theta \lambda}{\theta \lambda}\right|^{-m}\left(T_{N, m-1, \sigma}(\lambda)+\mathcal{A}(\varepsilon)\right)$ at two points with abscissa $a_{-}\left(\lambda^{*}, N, \varepsilon\right) \leq a_{+}\left(\lambda^{*}, N, \varepsilon\right)$ the interval

$$
I_{\varepsilon, N}:=\left[a_{-}\left(\lambda^{*}, N, \varepsilon\right), a_{+}\left(\lambda^{*}, N, \varepsilon\right)\right]
$$


and in particular $\lambda * \in I_{\varepsilon, N}$. Summarizing the above discussion, we arrive at the following lemma which is similar to that of [27] for Sturm-Liouville problems.

Lemma 3.3 For any eigenvalue $\lambda^{*}$, we can find $N_{0} \in \mathbb{Z}^{+}$and sufficiently small $\varepsilon$ such that $\lambda * \in I_{\varepsilon, N}$ for $N>N_{0}$. Moreover

$$
\left[a_{-}\left(\lambda^{*}, N, \varepsilon\right), a_{+}\left(\lambda^{*}, N, \varepsilon\right)\right] \rightarrow\left\{\lambda^{*}\right\} \text { as } N \rightarrow \infty \text { and } \varepsilon \rightarrow 0 .
$$

Proof. Since all eigenvalues of (15)-(19) are simple, then for large $N$ and sufficiently small $\varepsilon$ we have $\frac{\partial}{\partial \lambda}\left(\mathcal{K}(\lambda)+\left(\frac{\sin \theta \lambda}{\theta \lambda}\right)^{-m} \tilde{\mathcal{G}}_{\theta, m, N}(\lambda)\right)>0$, in a neighborhood of $\lambda^{*}$. Choose $N_{0}$ such that

$$
\mathcal{K}(\lambda)+\left(\frac{\sin \theta \lambda}{\theta \lambda}\right)^{-m} \tilde{\mathcal{G}}_{\theta, m, N_{0}}(\lambda)= \pm\left|\frac{\sin \theta \lambda}{\theta \lambda}\right|^{-m}\left(T_{N_{0}, m-1, \sigma}(\lambda)+\mathcal{A}(\varepsilon)\right)
$$

has two distinct solutions which we denote by $a_{-}\left(\lambda^{*}, N_{0}, \varepsilon\right) \leq a_{+}\left(\lambda^{*}, N_{0}, \varepsilon\right)$. The decay of $T_{N, m-1, \sigma}(\lambda) \rightarrow 0$ as $N \rightarrow \infty$ and $\mathcal{A}(\varepsilon) \rightarrow 0$ as $\varepsilon \rightarrow 0$ will ensure the existence of the solutions $a_{-}\left(\lambda^{*}, N, \varepsilon\right)$ and $a_{+}\left(\lambda^{*}, N, \varepsilon\right)$ as $N \rightarrow \infty$ and $\varepsilon \rightarrow 0$. For the second point we recall that $\tilde{\mathcal{G}}_{\theta, m, N}(\lambda) \rightarrow \mathcal{G}_{\theta, m}(\lambda)$ as $N \rightarrow \infty$ and as $\varepsilon \rightarrow 0$. Hence by taking the limit we obtain

$$
\begin{aligned}
& \mathcal{K}\left(a_{+}\left(\lambda^{*}, \infty, 0\right)\right)+\left(\frac{\sin \theta \lambda^{*}}{\theta \lambda^{*}}\right)^{-m} \mathcal{G}_{\theta, m}\left(a_{+}\left(\lambda^{*}, \infty, 0\right)\right)=0, \\
& \mathcal{K}\left(a_{-}\left(\lambda^{*}, \infty, 0\right)\right)+\left(\frac{\sin \theta \lambda^{*}}{\theta \lambda^{*}}\right)^{-m} \mathcal{G}_{\theta, m}\left(a_{-}\left(\lambda^{*}, \infty, 0\right)\right)=0,
\end{aligned}
$$

that is $\Omega\left(a_{+}\right)=\Omega\left(a_{-}\right)=0$. This leads us to conclude that $a_{+}=a_{-}=\lambda^{*}$, since $\lambda^{*}$ is a simple root.

$$
\begin{aligned}
& \text { Let } \tilde{\Omega}_{N}(\lambda):=\mathcal{K}(\lambda)+\left(\frac{\sin \theta \lambda}{\theta \lambda}\right)^{-m} \tilde{\mathcal{G}}_{\theta, m, N}(\lambda) \text {. Then (75) and (79) imply } \\
& \left|\Omega(\lambda)-\tilde{\Omega}_{N}(\lambda)\right| \leq\left|\frac{\sin \theta \lambda}{\theta \lambda}\right|^{-m}\left(T_{N, m-1, \sigma}(\lambda)+\mathcal{A}(\varepsilon)\right), \quad|\lambda|<\frac{N \pi}{\sigma}
\end{aligned}
$$

and $\theta$ is chosen sufficiently small for which $|\theta \lambda|<\pi$. Let $\lambda^{*}$ be an eigenvalue and $\lambda_{N}$ be its approximation. Thus $\Omega\left(\lambda^{*}\right)=0$ and $\tilde{\Omega}_{N}\left(\lambda_{N}\right)=0$. From (85) we have $\left|\tilde{\Omega}_{N}\left(\lambda^{*}\right)\right| \leq\left|\frac{\sin \theta \lambda^{*}}{\theta \lambda^{*}}\right|^{-m}\left(T_{N, m-1, \sigma}\left(\lambda^{*}\right)+\mathcal{A}(\varepsilon)\right)$. Now we estimate the error $\left|\lambda^{*}-\lambda_{N}\right|$, for the eigenvalue $\lambda^{*}$.

Lemma 3.4 Let $\lambda^{*}$ be an eigenvalue of (15)-(19). For sufficient large $N$ we have the following estimate

$$
\left|\lambda^{*}-\lambda_{N}\right|<\left|\frac{\sin \theta \lambda_{N}}{\theta \lambda_{N}}\right|^{-m} \frac{T_{N, m-1, \sigma}\left(\lambda_{N}\right)+\mathcal{A}(\varepsilon)}{\inf _{\zeta \in I_{\varepsilon, N}}\left|\Omega^{\prime}(\zeta)\right|} .
$$

Proof. Since $\Omega\left(\lambda_{N}\right)-\tilde{\Omega}_{N}\left(\lambda_{N}\right)=\Omega\left(\lambda_{N}\right)-\Omega\left(\lambda^{*}\right)$, then from (85) and after replacing $\lambda$ by $\lambda_{N}$ we obtain 


$$
\left|\Omega\left(\lambda_{N}\right)-\Omega\left(\lambda^{*}\right)\right| \leq\left|\frac{\sin \theta \lambda_{N}}{\theta \lambda_{N}}\right|^{-m}\left(T_{N, m-1, \sigma}\left(\lambda_{N}\right)+\mathcal{A}(\varepsilon)\right) .
$$

Using the mean value theorem yields that for some $\zeta \in J_{\varepsilon, N}:=\left[\min \left(\lambda^{*}, \lambda_{N}\right), \max \left(\lambda^{*}\right.\right.$, $\left.\lambda_{N}\right)$,

$$
\left|\left(\lambda^{*}-\lambda_{N}\right) \Omega^{\prime}(\zeta)\right| \leq\left|\frac{\sin \theta \lambda_{N}}{\theta \lambda_{N}}\right|^{-m}\left(T_{N, m-1, \sigma}\left(\lambda_{N}\right)+\mathcal{A}(\varepsilon)\right), \quad \zeta \in J_{\varepsilon, N} \subset I_{\varepsilon, N} .
$$

Since the eigenvalues are simple, then for sufficiently large $N \inf _{\zeta \in I_{\varepsilon, N}}\left|\Omega^{\prime}(\zeta)\right|>0$ and we get (86).

\section{Numerical examples}

This section includes two detailed worked examples illustrating the above technique. By $E_{S}$ and $E_{H}$ we mean the absolute errors associated with the results of the classical sinc method and our new method (Hermite interpolations) respectively. All examples are computed in [22] with the classical sinc method. We indicate in these examples the effect of the amplitude error in the method by determining enclosure intervals for different values of $\varepsilon$. We also indicate the effect of the parameters $m$ and $\theta$ by several choices. Every example is accompanied with six figures illustrating $\Omega(\lambda), \tilde{\Omega}(\lambda)$ and the enclosure curves dominating the zeros. Recall that $\mathrm{a}_{ \pm}(\lambda)$ are defined by

$$
a \pm(\lambda)=\Omega_{N}(\lambda) \pm\left|\frac{\sin \theta \lambda}{\theta \lambda}\right|^{-m}\left(T_{N, m-1, \sigma}(\lambda)+\mathcal{A}(\varepsilon)\right), \quad|\lambda|<\frac{N_{\pi}}{\sigma} .
$$

Recall also that the enclosure interval $I_{\varepsilon, N}=\left[a_{-}, a_{+}\right]$is determined by solving

$$
a_{ \pm}(\lambda)=0,|\lambda|<\frac{N \pi}{\sigma} .
$$

Example 1 Consider the system

$$
\begin{aligned}
& u_{2}^{\prime}(x)-r(x) u_{1}(x)=\lambda u_{1}(x), \quad u_{1}^{\prime}(x)+r(x) u_{2}(x)=-\lambda u_{2}(x), \quad x \in[-1,0) \cup(0,1], \\
& u_{1}(-1)+u_{2}(-1)=0, \quad u_{1}(1)+u_{2}(1)=0, \\
& u_{1}\left(0^{-}\right)-2 u_{1}\left(0^{+}\right)=0, \quad u_{2}\left(0^{-}\right)-2 u_{2}\left(0^{+}\right)=0 .
\end{aligned}
$$

Here

$$
r_{1}(x)=r_{2}(x)=r(x)= \begin{cases}x^{2}, & x \in[-1,0) \\ x, & (0,1]\end{cases}
$$

$\alpha=\beta=\frac{\pi}{4}$ and $\delta=2$. Direct calculations give

$$
\mathcal{K}(\lambda)=-2 \sin [2 \lambda]
$$

and

$$
\Omega(\lambda)=-2 \sin \left[\frac{5}{6}+2 \lambda\right],
$$


therefore the eigenvalues are $\lambda_{k}=\frac{6 k \pi-5}{12}, k \in \mathbb{Z}$. The following four tables indicate the application of our technique to this problem and the effect of $\varepsilon, \theta$ and $m$ (Tables 1 , 2, 3and 4).

In the following, the Figures 1and 2illustrate the comparison between $\Omega(\lambda)$ and $\tilde{\Omega}_{N}(\lambda)$ for different values of $m$ and $\theta$.

Figures 3 and 4, for $N=20, m=6$ and $\theta=1 / 7$, illustrate the enclosure intervals for $\varepsilon=10^{-8}$ and $\varepsilon=10^{-12}$ respectively.

Also, Figures 5 and 6illustrate the enclosure intervals for $\varepsilon=10^{-8}$ and $\varepsilon=10^{-12}$ respectively, but for $m=10, \theta=1 / 5$.

Table $1 N=20, m=6$, and $\theta=1 / 7$

\begin{tabular}{lllll}
\hline$\lambda_{\boldsymbol{k}}$ Sinc $\boldsymbol{\lambda}_{\boldsymbol{k}, \boldsymbol{N}}$ & Exact $\boldsymbol{\lambda}_{\boldsymbol{k}}$ & Hermite $\boldsymbol{\lambda}_{\boldsymbol{k}, \boldsymbol{N}}$ & $E_{\boldsymbol{S}}$ & $E_{H}$ \\
\hline$\lambda_{-2}-3.558259424986099$ & -3.5582593202564599 & -3.5582593202488253 & $1.047 \times 10^{-7}$ & $7.634 \times 10^{-12}$ \\
$\lambda_{-1}-1.9874629152275372$ & -1.9874629934615633 & -1.9874629934659096 & $7.823 \times 10^{-8}$ & $4.346 \times 10^{-12}$ \\
$\lambda_{0}-0.4166665411688605$ & -0.41666666666666667 & -0.41666666665625623 & $1.255 \times 10^{-7}$ & $1.041 \times 10^{-11}$ \\
$\lambda_{1} 1.1541296373132959$ & 1.1541296601282299 & 1.1541296601279385 & $2.281 \times 10^{-8}$ & $2.913 \times 10^{-13}$ \\
$\lambda_{2} 2.7249258102486835$ & 2.7249259869231266 & 2.7249259869365776 & $1.767 \times 10^{-7}$ & $1.345 \times 10^{-11}$ \\
\hline
\end{tabular}

Table $2 N=20, m=10$ and $\theta=1 / 5$

\begin{tabular}{lllll}
\hline$\lambda_{\boldsymbol{k}} \operatorname{Sinc} \lambda_{\boldsymbol{k}, \boldsymbol{N}}$ & Exact $\boldsymbol{\lambda}_{\boldsymbol{k}}$ & Hermite $\boldsymbol{\lambda}_{\boldsymbol{k}, \boldsymbol{N}}$ & $\boldsymbol{E}_{\boldsymbol{S}}$ & $\boldsymbol{E}_{\boldsymbol{H}}$ \\
\hline$\lambda_{-2}-3.558259320291224$ & -3.5582593202564599 & -3.5582593202564596 & $3.476 \times 10^{-11}$ & $4.441 \times 10^{-16}$ \\
$\lambda_{-1}-1.9874629934481942$ & -1.9874629934615633 & -1.9874629934615631 & $1.3369 \times 10^{-11}$ & $2.220 \times 10^{-16}$ \\
$\lambda_{0}-0.416666666672866$ & -0.4166666666666667 & -0.416666666666666635 & $6.199 \times 10^{-12}$ & $3.331 \times 10^{-16}$ \\
$\lambda_{1} 1.1541296601306603$ & 1.1541296601282299 & 1.1541296601282297 & $2.431 \times 10^{-12}$ & $2.220 \times 10^{-16}$ \\
$\lambda_{2} 2.724925986925671$ & 2.7249259869231266 & 2.7249259869231270 & $2.544 \times 10^{-12}$ & $4.440 \times 10^{-16}$ \\
\hline
\end{tabular}

Table 3 The approximation $\lambda_{k, N}$ and the exact solution $\lambda_{k}$ are all inside the interval [ $a_{-}$, $a_{+}$] for different values of $\varepsilon$, where $N=20, m=6, \theta=1 / 7$

\begin{tabular}{llll}
\hline$\lambda_{\boldsymbol{k}}$ Exact $\boldsymbol{\lambda}_{\boldsymbol{k}}$ & {$\left[\boldsymbol{a}_{-}, \boldsymbol{a}_{+}\right], \boldsymbol{\varepsilon}=\mathbf{1 0}^{-\mathbf{8}}$} & {$\left[\boldsymbol{a}_{-}, \boldsymbol{a}_{+}\right], \boldsymbol{\varepsilon}=\mathbf{1 0}^{-\mathbf{1 2}}$} & $\boldsymbol{\lambda}_{\boldsymbol{k}, \boldsymbol{N}}$ \\
\hline$\lambda_{-2}-3.5582593202564599$ & {$[-3.753326,-3.387861]$} & {$[-3.565657,-3.551497]$} & -3.5582593202488253 \\
$\lambda_{-1}-1.9874629934615633$ & {$[-2.134039,-1.835158]$} & {$[-1.991487,-1.983156]$} & -1.9874629934659096 \\
$\lambda_{0}-0.4166666666666667$ & {$[-0.562608,-0.277479]$} & {$[-0.427095,-0.406713]$} & -0.416666666665625623 \\
$\lambda_{1} 1.1541296601282299$ & {$[1.017775,1.295214]$} & {$[1.153811,1.154454]$} & 1.1541296601279385 \\
$\lambda_{2} 2.7249259869231266$ & {$[2.561929,2.895812]$} & {$[2.711312,2.738729]$} & 2.7249259869365776 \\
\hline
\end{tabular}

$E_{5}\left(\mathcal{G}_{\theta, m}\right)=1.7659 \times 10^{6}, E_{4}\left(\mathcal{G}_{\theta, m}\right)=278897, \ell=1, M_{\mathcal{G}_{\theta, m}}=4563.57$

Table 4 For $N=20, m=10$ and $\theta=1 / 5, \lambda_{k, N}$ and $\lambda_{k}$ are all inside the interval $\left[a_{-}, a_{+}\right]$for different values of $\varepsilon$

\begin{tabular}{llll}
\hline$\lambda_{\boldsymbol{k}}$ Exact $\lambda_{\boldsymbol{k}}$ & {$\left[\boldsymbol{a}_{-}, \boldsymbol{a}_{+}\right], \varepsilon=1 \mathbf{0}^{-\mathbf{8}}$} & {$\left[\boldsymbol{a}_{-}, \boldsymbol{a}_{+}\right], \varepsilon=1 \mathbf{0}^{-12}$} & $\boldsymbol{\lambda}_{\boldsymbol{k}, \boldsymbol{N}}$ \\
\hline$\lambda_{-2}-3.5582593202564599$ & {$[-3.739980,-3.404715]$} & {$[-3.560364,-3.556152]$} & -3.5582593202564596 \\
$\lambda_{-1}-1.9874629934615633$ & {$[-2.080163,-1.899068]$} & {$[-1.988611,-1.986313]$} & -1.9874629934615631 \\
$\lambda_{0}-0.4166666666666667$ & {$[-0.487002,-0.346801]$} & {$[0.417553,-0.415779]$} & -0.41666666666666635 \\
$\lambda_{1} 1.1541296601282299$ & {$[1.079274,1.230862]$} & {$[1.153170,1.155090]$} & 1.1541296601282297 \\
$\lambda_{2} 2.7249259869231266$ & {$[2.614866,2.845166]$} & {$[2.723467,2.726388]$} & 2.7249259869231270 \\
\hline$E_{9}\left(\mathcal{G}_{\theta, m}\right)=1.15498 \times 10^{9}, E_{8}\left(\mathcal{G}_{\theta, m}\right)=2.44207 \times 10^{8}, \ell=1, M_{\mathcal{G}_{\theta, m}}=20378$
\end{tabular}




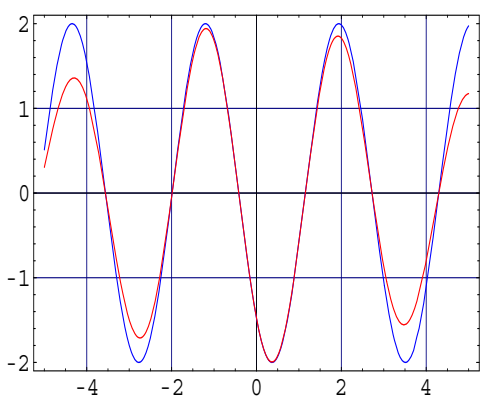

Figure $1 \Omega(\lambda), \tilde{\Omega}_{N}(\lambda)$ with $N=20, m=6$ and $\theta=1 / 7$.

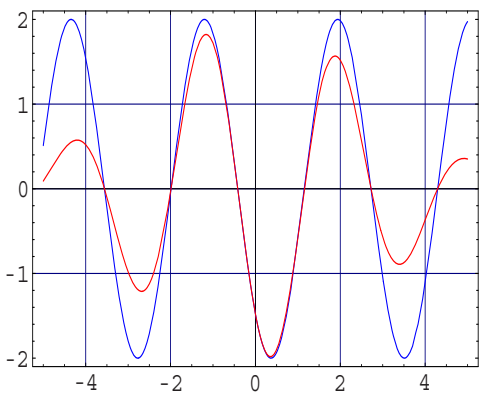

Figure $2 \Omega(\lambda), \tilde{\Omega}_{N}(\lambda)$ with $N=20, m=10$ and $\theta=1 / 5$

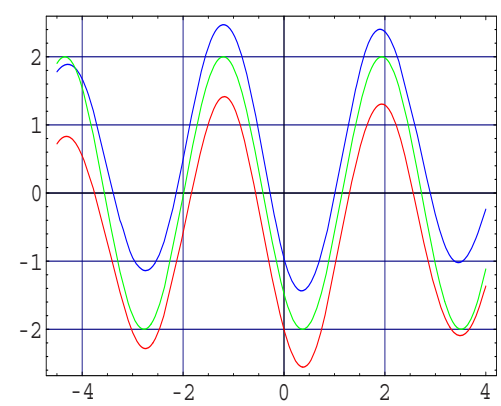

Figure $3 a_{+}, \Omega(\lambda)$, $a_{\text {. with }} N=20, m=6, \theta=1 / 7$ and $\varepsilon=10^{-8}$

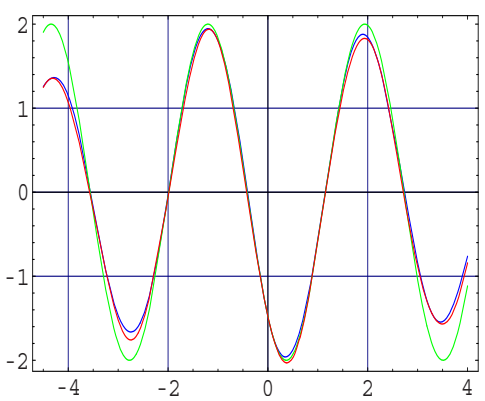

Figure $4 a_{+}, \Omega(\lambda), a_{-}$with $N=20, m=6, \theta=1 / 7$ and $\varepsilon=10^{-12}$. 


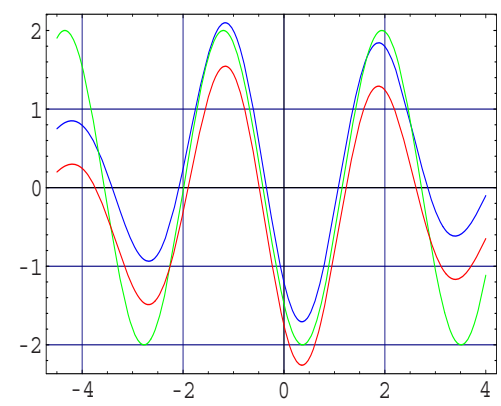

Figure $5 a_{+}, \Omega(\lambda), a_{-}$with $N=20, m=10, \theta=1 / 5$ and $\varepsilon=10^{-8}$

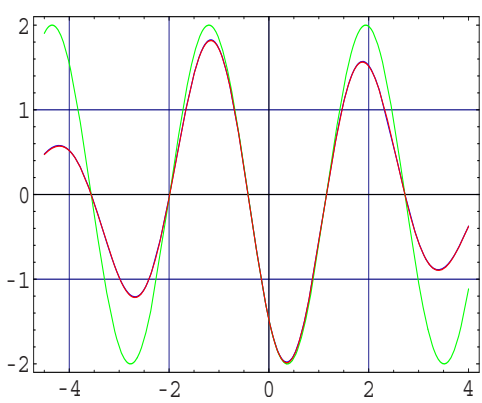

Figure $6 a_{+}, \Omega(\lambda), a_{-}$with $N=20, m=10, \theta=1 / 5$ and $\varepsilon=10^{-12}$

Example 2 In this example we consider the system

$$
\begin{aligned}
& u_{2}^{\prime}(x)-r(x) u_{1}(x)=\lambda u_{1}(x), \quad u_{1}^{\prime}(x)+r(x) u_{2}(x)=-\lambda u_{2}(x), \quad x \in[-1,0) \cup(0,1], \\
& \sqrt{3} u_{1}(-1)+u_{2}(-1)=0, \quad u_{1}(1)+\sqrt{3} u_{2}(1)=0, \\
& u_{1}\left(0^{-}\right)-3 u_{1}\left(0^{+}\right)=0, \quad u_{2}\left(0^{-}\right)-3 u_{2}\left(0^{+}\right)=0,
\end{aligned}
$$

where

$$
r_{1}(x)=r_{2}(x)=r(x)= \begin{cases}x, & x \in[-1,0) \\ x^{2}+1, & (0,1],\end{cases}
$$

$\alpha=\frac{\pi}{3}, \beta=\frac{\pi}{6}$ and $\delta=3$. Direct calculations give

$$
\mathcal{K}(\lambda)=3 \sin \left[\frac{\pi}{6}-2 \lambda\right]
$$

and

$$
\Omega(\lambda)=\frac{3}{2}\left(\cos \left[\frac{5}{6}+2 \lambda\right]-\sqrt{3} \sin \left[\frac{5}{6}+2 \lambda\right]\right),
$$

therefore the eigenvalues are $\lambda_{k}=\frac{(6 k+1) \pi-5}{12}, k \in \mathbb{Z}$. As in the previous example, Figures 7, 8, 9, 10, 11and 12illustrate the results of Tables 5, 6, 7and 8. Also, Figures 5and 6illustrate the enclosure intervals for $\varepsilon=10^{-8}$ and $\varepsilon=10^{-12}$ respectively, but for $m=9, \theta=2 / 11$. 


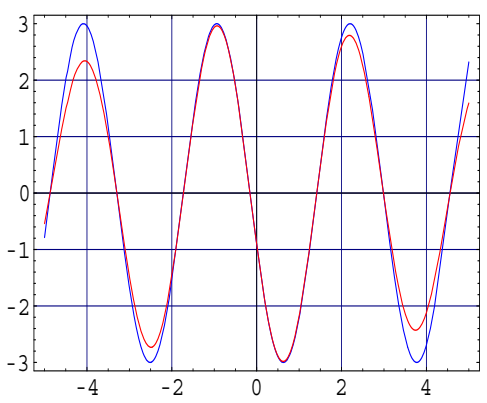

Figure $7 \Omega(\lambda), \tilde{\Omega}_{N}(\lambda)$ with $N=20, m=5$ and $\theta=2 / 15$

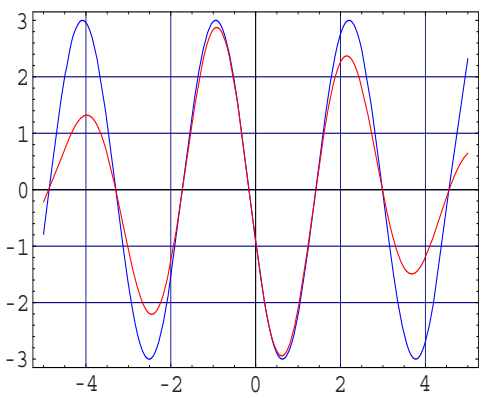

Figure $8 \Omega(\lambda), \tilde{\Omega}_{N}(\lambda)$ with $N=20, m=9$ and $\theta=2 / 11$

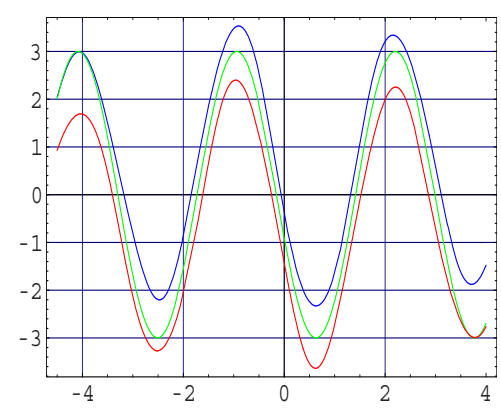

Figure $9 a_{+} \Omega(\lambda), a_{-}$with $N=20, m=5, \theta=2 / 15$ and $\varepsilon=10^{-8}$.

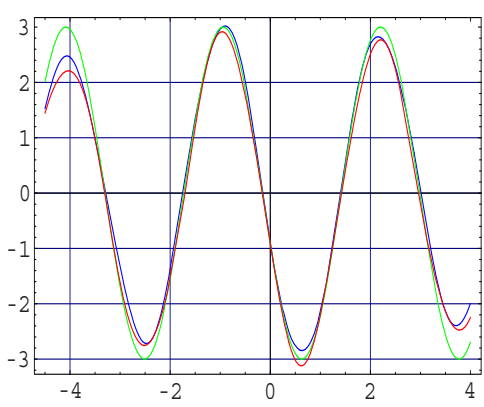

Figure $10 a_{+}, \Omega(\lambda), a_{-}$with $N=20, m=5, \theta=2 / 15$ and $\varepsilon=10^{-12}$. 


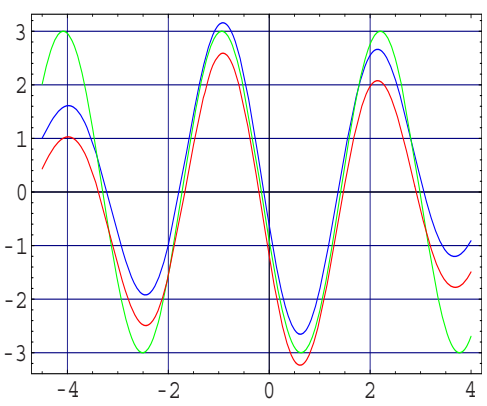

Figure $11 a_{+}, \Omega(\lambda), a_{-}$with $N=20, m=9, \theta=2 / 11$ and $\varepsilon=10^{-8}$.

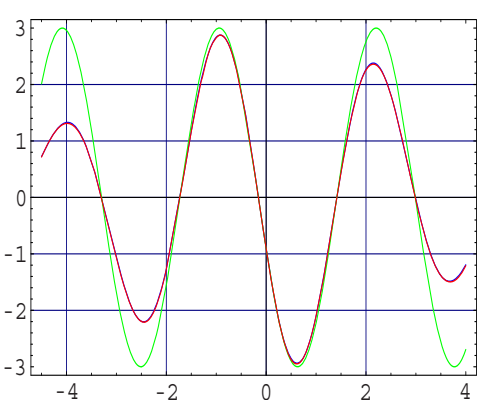

Figure $12 a_{+}, \Omega(\lambda), a_{-}$with $N=20, m=9, \theta=2 / 11$ and $\varepsilon=10^{-12}$

Table $5 N=20, m=5, \theta=2 / 15$

\begin{tabular}{lllll}
\hline$\lambda_{\boldsymbol{k}}$ Sinc $\boldsymbol{\lambda}_{\boldsymbol{k}, \boldsymbol{N}}$ & Exact $\boldsymbol{\lambda}_{\boldsymbol{k}}$ & Hermite $\boldsymbol{\lambda}_{\boldsymbol{k}, \boldsymbol{N}}$ & $E_{\boldsymbol{S}}$ & $E_{H}$ \\
\hline$\lambda_{-2}-3.2964601812375145$ & -3.2964599324573105 & -3.2964599325093795 & $2.488 \times 10^{-7}$ & $5.207 \times 10^{-11}$ \\
$\lambda_{-1}-1.725663818527078$ & -1.725663605662414 & -1.7256636055362218 & $2.129 \times 10^{-7}$ & $1.262 \times 10^{-10}$ \\
$\lambda_{0}-0.15486730070281368$ & -0.15486727886751722 & -0.15486727888681906 & $2.183 \times 10^{-8}$ & $1.930 \times 10^{-11}$ \\
$\lambda_{1} 1.4159289890168527$ & 1.4159290479273794 & 1.4159290479703164 & $5.891 \times 10^{-8}$ & $4.294 \times 10^{-11}$ \\
$\lambda_{2} 2.9867250912171013$ & 2.986725374722276 & 2.9867253745880475 & $2.835 \times 10^{-7}$ & $1.342 \times 10^{-10}$ \\
\hline
\end{tabular}

Table $6 N=20, m=9, \theta=2 / 11$

\begin{tabular}{lllll}
\hline$\lambda_{\boldsymbol{k}}$ Sinc $\boldsymbol{\lambda}_{\boldsymbol{k}, \boldsymbol{N}}$ & Exact $\boldsymbol{\lambda}_{\boldsymbol{k}}$ & Hermite $\boldsymbol{\lambda}_{\boldsymbol{k}, \boldsymbol{N}}$ & $\boldsymbol{E}_{\boldsymbol{S}}$ & $\boldsymbol{E}_{\boldsymbol{H}}$ \\
\hline$\lambda_{-2}-3.2964599325179624$ & -3.2964599324573105 & -3.2964599324573127 & $6.065 \times 10^{-11}$ & $2.220 \times 10^{-15}$ \\
$\lambda_{-1}-1.7256636056618686$ & -1.725663605662414 & -1.725663605662413 & $5.453 \times 10^{-13}$ & $8.881 \times 10^{-16}$ \\
$\lambda_{0}-0.1548672788373793$ & -0.15486727886751722 & -0.1548672788675186 & $3.013 \times 10^{-11}$ & $1.388 \times 10^{-15}$ \\
$\lambda_{1} 1.415929047873631$ & 1.4159290479273794 & 1.4159290479273827 & $5.374 \times 10^{-11}$ & $3.331 \times 10^{-15}$ \\
$\lambda_{2} 2.986725374801227$ & 2.986725374722276 & 2.9867253747222686 & $7.895 \times 10^{-11}$ & $7.549 \times 10^{-15}$ \\
\hline
\end{tabular}

Table 7 For $N=20, m=5$ and $\theta=2 / 15$, the approximation $\lambda_{k, N}$ and the exact solution $\lambda_{k}$ are all inside the interval $\left[a_{-}, a_{+}\right]$for different values of $\varepsilon$

\begin{tabular}{llll}
\hline$\lambda_{\boldsymbol{k}}$ Exact $\boldsymbol{\lambda}_{\boldsymbol{k}}$ & {$\left[\boldsymbol{a}_{-}, \boldsymbol{a}_{+}\right], \boldsymbol{\varepsilon}=\mathbf{1 0}^{-\mathbf{5}}$} & {$\left[\boldsymbol{a}_{-}, \boldsymbol{a}_{+}\right], \boldsymbol{\varepsilon}=\mathbf{1 0}^{-\mathbf{1 0}}$} & $\boldsymbol{\lambda}_{\boldsymbol{k}, \boldsymbol{N}}$ \\
\hline$\lambda_{-2}-3.2964599324573105$ & {$[-3.402745,-3.177441]$} & {$[-3.305475,-3.286114]$} & -3.2964599325093795 \\
$\lambda_{-1}-1.725663605662414$ & {$[-1.840530,-1.615062]$} & {$[-1.749858,-1.702197]$} & -1.7256636055362218 \\
$\lambda_{0}-0.15486727886751722$ & {$[-0.250393,-0.067671]$} & {$[-0.158786,-0.151287]$} & -0.15486727888681906 \\
$\lambda_{1} 1.4159290479273794$ & {$[1.323736,1.520703]$} & {$[1.408023,1.424863]$} & 1.4159290479703164 \\
$\lambda_{2} 2.986725374722276$ & {$[2.863300,3.109311]$} & {$[2.960260,3.012514]$} & 2.9867253745880475 \\
\hline
\end{tabular}

$E_{4}\left(\mathcal{G}_{\theta, m}\right)=319577, E_{3}\left(\mathcal{G}_{\theta, m}\right)=48315.5, \ell=1, M_{\mathcal{G}_{\theta, m}}=4400.77$ 
Table 8 With $N=20, m=9$ and $\theta=2 / 11, \lambda_{k, N}$ and $\lambda_{k}$ are all inside the interval $\left[a_{-}, a_{+}\right]$ for different values of $\varepsilon$

\begin{tabular}{llll}
\hline$\lambda_{\boldsymbol{k}}$ Exact $\boldsymbol{\lambda}_{\boldsymbol{k}}$ & {$\left[\boldsymbol{a}_{-,} \boldsymbol{a}_{+}\right], \varepsilon=1 \mathbf{0}^{-\mathbf{5}}$} & {$\left[\boldsymbol{a}_{-}, \boldsymbol{a}_{+}\right], \varepsilon=1 \mathbf{0}^{-\mathbf{1 0}}$} & $\boldsymbol{\lambda}_{\boldsymbol{k}, \boldsymbol{N}}$ \\
\hline$\lambda_{-2}-3.2964599324573105$ & {$[-3.381001,-3.215245]$} & {$[-3.297352,-3.295553]$} & -3.2964599324573127 \\
$\lambda_{-1}-1.725663605662414$ & {$[-1.781207,-1.671159]$} & {$[-1.725760,-1.725566]$} & -1.725663605662413 \\
$\lambda_{0}-0.15486727886751722$ & {$[-0.202971,-0.107279]$} & {$[-0.155364,-0.154374]$} & -0.1548672788675186 \\
$\lambda_{1} 1.4159290479273794$ & {$[1.362486,1.469718]$} & {$[1.414521,1.417324]$} & 1.4159290479273827 \\
$\lambda_{2} 2.986725374722276$ & {$[2.912282,3.065032]$} & {$[2.984366,2.989098]$} & 2.9867253747222686 \\
\hline
\end{tabular}

$E_{8}\left(\mathcal{G}_{\theta, m}\right)=4.61204 \times 10^{8}, E_{7}\left(\mathcal{G}_{\theta, m}\right)=8.92709 \times 10^{7}, \ell=1, M_{\mathcal{G}_{\theta, m}}=20713.1$

\section{Acknowledgements}

This article was funded by the Deanship of Scientific Research (DSR), King Abdulaziz University, Jeddah. The authors, therefore, acknowledge with thanks DSR technical and financial support.

\section{Author details}

${ }^{1}$ Department of Mathematics, Faculty of Science, King Abdulaziz University, Jeddah, Saudi Arabia ${ }^{2}$ Department of Mathematics, Faculty of Science, Beni-Suef University, Beni-Suef, Egypt

\section{Authors' contributions}

The authors have equal contributions to each part of this article. All the authors read and approved the final manuscript.

\section{Competing interests}

The authors declare that they have no competing interests.

Received: 20 March 2012 Accepted: 10 May 2012 Published: 10 May 2012

\section{References}

1. Grozev, GR, Rahman, Ql: Reconstruction of entire functions from irregularly spaced sample points. Canad J Math. 48 777-793 (1996). doi:10.4153/CJM-1996-040-7

2. Higgins, JR, Schmeisser, G, Voss, JJ: The sampling theorem and several equivalent results in analysis. J Comput Anal Appl. 2, 333-371 (2000)

3. Hinsen, G: Irregular sampling of bandlimited LP-functions. J Approx Theory. 72, 346-364 (1993). doi:10.1006/ jath.1993.1027

4. Jagerman, D, Fogel, L: Some general aspects of the sampling theorem. IRE Trans Inf Theory. 2, 139-146 (1956). doi:10.1109/TIT.1956.1056821

5. Annaby, MH, Asharabi, RM: Error analysis associated with uniform Hermite interpolations of bandlimited functions. J Korean Math Soc. 47, 1299-1316 (2010). doi:10.4134/JKMS.2010.47.6.1299

6. Higgins, JR: Sampling Theory in Fourier and Signal Analysis: Foundations. Oxford University Press, Oxford (1996)

7. Butzer, PL, Schmeisser, G, Stens, RL: An introduction to sampling analysis. pp. 17-121. Kluwer, New York (2001) Non Uniform Sampling: Theory and Practices

8. Butzer, PL, Higgins, JR, Stens, RL: Sampling theory of signal analysis. Development of Mathematics 1950-2000. pp. 193234.Birkhäuser, Basel, Switzerland (2000)

9. Annaby, MH, Asharabi, RM: On sinc-based method in computing eigenvalues of boundary-value problems. SIAM J Numer Anal. 46, 671-690 (2008). doi:10.1137/060664653

10. Annaby, MH, Tharwat, MM: On computing eigenvalues of second-order linear pencils. IMA J Numer Anal. 27, 366-380 (2007)

11. Annaby, MH, Tharwat, MM: Sinc-based computations of eigenvalues of Dirac systems. BIT. 47, 699-713 (2007) doi:10.1007/s10543-007-0154-8

12. Annaby, MH, Tharwat, MM: On the computation of the eigenvalues of Dirac systems. (2011) Calcolo, doi:10.1007/ s10092-011-0052-y

13. Boumenir, A, Chanane, B: Eigenvalues of S-L systems using sampling theory. Appl Anal. 62, 323-334 (1996). doi:10.1080/ 00036819608840486

14. Lund, J, Bowers, K: Sinc Methods for Quadrature and Differential Equations. SIAM, Philadelphia, PA (1992)

15. Stenger, F: Numerical methods based on Whittaker cardinal, or sinc functions. SIAM Rev. 23, 156-224 (1981)

16. Stenger, F: Numerical Methods Based on Sinc and Analytic Functions. Springer-Verlag, New York (1993)

17. Butzer, PL, Splettstösser, W, Stens, RL: The sampling theorem and linear prediction in signal analysis. Jahresber Deutsch Math-Verein. 90, 1-70 (1988)

18. Jagerman, D: Bounds for truncation error of the sampling expansion. SIAM J Appl Math. 14, 714-723 (1966). doi:10.1137/0114060

19. Boas, RP: Entire Functions. Academic Press, New York (1954)

20. Annaby, MH, Asharabi, RM: Approximating eigenvalues of discontinuous problems by sampling theorems. J Numer Math. 16, 163-183 (2008)

21. Chanane, B: Eigenvalues of Sturm Liouville problems with discontinuity conditions inside a finite interval. Appl Math Comput. 188, 1725-1732 (2007). doi:10.1016/j.amc.2006.11.082 
22. Tharwat, MM, Bhrawy, AH, Yildirim, A: Numerical Computation of Eigenvalues of Discontinuous Dirac System Using Sinc Method with Error Analysis. International Journal of Computer Mathematics 2012, 20 (2012). Article ID GCOM-20120079-B

23. Levitan, BM, Sargsjan, IS: Introduction to Spectral Theory: Self Adjoint Ordinary Differential Operators. In Translation of Mthematical Monographs, vol. 39,American Mathematical Society, Providence, RI (1975)

24. Tharwat, MM: Discontinuous Sturm-Liouville problems and associated sampling theories. Abstr Appl Anal 2011, 1-30 (2011). doi:10.1155/2011/610232

25. Levitan, BM, Sargsjan, IS: Sturm-Liouville and Dirac Operators. Kluwer Acadamic, Dordrecht (1991)

26. Chadan, K, Sabatier, PC: Inverse Problems in Quantum Scattering Theory. Springer-Verlag, Berlin, 2 (1989)

27. Boumenir, A: Higher approximation of eigenvalues by the sampling method. BIT. 40, 215-225 (2000). doi:10.1023/ A:1022334806027

doi:10.1186/1687-1847-2012-59

Cite this article as: Tharwat and Bhrawy: Computation of eigenvalues of discontinuous dirac system using

Hermite interpolation technique. Advances in Difference Equations 2012 2012:59.

\section{Submit your manuscript to a SpringerOpen ${ }^{\circ}$} journal and benefit from:

- Convenient online submission

- Rigorous peer review

- Immediate publication on acceptance

- Open access: articles freely available online

- High visibility within the field

- Retaining the copyright to your article

Submit your next manuscript at $\gg$ springeropen.com 